\title{
Le contrat social haïtien
}

\section{Carlo Avierl Célius}

\section{OpenEdition}

\section{Journals}

Édition électronique

URL : http://journals.openedition.org/plc/542

DOI : $10.4000 /$ plc.542

ISSN : 2117-5209

\section{Éditeur}

L'Harmattan

Édition imprimée

Date de publication : 1 janvier 1998

Pagination : 27-70

ISSN : 1279-8657

\section{Référence électronique}

Carlo Avierl Célius, « Le contrat social haïtien », Pouvoirs dans la Caraïbe [En ligne], 10 | 1998, mis en ligne le 14 mars 2011, consulté le 19 avril 2019. URL : http://journals.openedition.org/plc/542 ; DOI : $10.4000 /$ plc.542 


\title{
Le contrat social haïtien
}

\author{
par Carlo Avierl CELIUS \\ Doctorant \\ Ecole des Hautes Etudes en Sciences Sociales \\ Paris*
}

\section{NEGOCIER OU RENEGOCIER \\ LE CONTRAT SOCIAL HAÏTIEN?}

Dans sa contribution au colloque international de Port-au-Prince consacré aux Transitions Démocratiques, l'anthropologue MichelRolph Trouillot a affirmé qu'il n'y a jamais eu de contrat social haïtien. Selon lui "l'établissement" d'un tel pacte est la condition même de l'instauration de la démocratie en Haïti ${ }^{1}$. Trouillot poursuit son plaidoyer pour ce qu'il considère comme la question fondamentale haïtienne : la réconciliation de l'Etat avec la Nation qui suppose une participation effective de la paysannerie à la chose publique ${ }^{2}$. La cause défendue n'est pas ici en question. Mais la thèse de l'inexistence d'un contrat soulève quelques interrogations d'ordre historique.

*. Je remercie pour leur précieux concours Nathalie Brisson Lamaute, Myriam Cottias, Fritz Pierre et Laurent Dubois.

1. Michel-Rolph Trouillot, "Démocratie et société civile", in Laënnec Hurbon (sous la direction de), Les transitions démocratiques. Actes du colloque international de Port-au-Prince, Haïti. Paris, Syros, 1996, pp.225-231.

2 . Présentant son ouvrage Les racines historiques de l'état duvaliérien, Port-auPrince, Editions Deschamps, 1986, l'auteur explique que "La résolution de la crise [haïtienne] ne peut être un projet individuel : elle exige une mobilisation nationale. Mais cette mobilisation est impossible sans la participation effective de la paysannerie à la chose publique. En peu de mots, cette analyse est un plaidoyer pour la réconciliation de l'Etat et de la Nation." pp.11- 12 
Avant tout, il convient de remarquer que cette thèse est à la fois une approbation et un désaveu. Elle relève d'une lecture de l'histoire politique à partir de la théorie du contrat, en même temps elle désapprouve le principe qui fonde celle-ci, à savoir que toute organisation sociale se construit sur une base contractuelle. Cette loi n'est pas une nécessité absolue parce que la société haïtienne y échappe. Cependant son inexistence entraîne un fâcheux dysfonctionnement : elle disjoint l'Etat de la Nation. Ainsi une société peut ne pas s'édifier sur un contrat, mais cette convention est nécessaire à son bon fonctionnement ${ }^{3}$.

Comment naît un contrat, selon Trouillot? Ou plutôt comment naîtra le contrat haïtien ? "La transition vers la démocratie, explique-til, ne sera possible que dans la mesure où l'État haïtien, la bourgeoisie et les classes moyennes qui demeurent soudées à cet État offrent à la majorité de la population de ce pays, qui demeure paysanne, les moyens de se constituer institutionnellement. Les gains de la société civile haïtienne se mesurent finalement à partir de cet espace." L'auteur ajoute : "La démocratie haïtienne exige donc l'établissement d'un contrat social - c'est-à-dire, la participation de la majorité haïtienne dans les choix qui influencent la destinée du pays. L'inclusion de la majorité exige à son tour la reconnaissance par les élites urbaines et leurs partenaires étrangers du fait qu'Haïti demeure fondamentalement un pays de paysans pauvres ${ }^{4}$ ". Il appartient aux autres catégories sociales de "reconnaître" la paysannerie et de 1" inclure" dans les pratiques du pouvoir. Au fondement du contrat donc une "volonté de communication" ou le principe de

${ }^{3}$. Le contrat est-il perçu ici comme un "principe régulateur" et non constitutif du politique ? Il serait alors d'ordre normatif. Ce qui ne semble pas être le cas dans l'approche de Trouillot. Faut-il comprendre celle-ci dans la perspective du renouveau du contrat (E. Faure, Pour un nouveau contrat social, 1973 ; J. Rawls, Theory of justice 1971) ? Mais là il n'est pas un concept opératoire qui rend compte de la société civile. Selon Rawls il n'explique pas l'institution et encore moins l'organisation constitutionnelle de la société politique. Voir "Le contrat social" in André-Jean Arnaud (sous la dir.), Dictionnaire Encyclopédique de théorie et de sociologie du

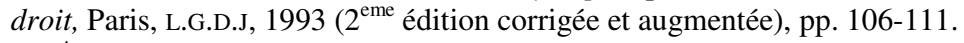

4. C'est moi qui souligne. Cette indication sera désormais signalée par un astérisque $(*)$. 
"concertation". volontaire, unilatéral. Or, les groupes sociaux dominants n'acceptent pas de changer les relations de pouvoir sans en être contraints par une modification des rapports de force. Cela signifie qu'un nouveau mode d'organisation sociale est possible si l'état des rapports de force le permet, c'est-à-dire si celui-ci crée les conditions de négociations entre les groupes sociaux antagonistes.

Le contrat, sur la base de ces considérations, peut se comprendre comme la formalisation d'un modèle de société négocié entre des forces politiques à un moment donné. Cette proposition ne surmonte certainement pas les ambiguïtés de la théorie du contrat ${ }^{6}$. Elle offre un angle d'approche, certes limité, mais apparemment fructueux, de la question de la formation sociale haïtienne.

Je soutiendrai donc qu'à un moment de l'histoire d'Haïti, le jeu des forces politiques a conduit à des négociations entre les groupes sociaux. Il en est résulté un contrat formalisant un modèle social à partir duquel s'est structurée la société haïtienne ${ }^{7}$. Ce pacte n'a jamais été renégocié, malgré certaines remises en cause. L'affirmation de son inexistence doit beaucoup, sans doute, au fait qu'il soit antérieur à

5 . Ici Trouillot semble s'inscrire dans le schéma du contrat proposé par E. Faure. Selon celui-ci le nouveau contrat " doit s'élaborer autour du thème de la concertation qui correspond à la reconnaissance de la responsabilité communautaire aussi bien parmi les gouvernés que parmi les gouvernants. Ainsi, le contrat social, qui fut, selon Rousseau, " un contrat de sécurité ", deviendra un "contrat de promotion " où chacun sera ouvert aux autres de sorte qu'il soit tenu compte des conditions de travail, de la typologie sociale, des structures économiques, de la qualité de la vie ...", André-Jean Arnaud, op. cit., p.110.

6. Sur la controverse autour du contrat social et les ambiguïté inhérentes à la théorie du contrat, se référer à la présentation synthétique et aux commentaires $d u$ Dictionnaire encyclopédique de théorie et de sociologie du droit..., op. cit., pp. 106 111.

7. Certains auteurs ont supposé l'existence de ce contrat, sans y consacrer une étude spécifique. Roger Dorsinville, par exemple, parlant du mouvement politique de 1946, s'est demandé : “... derrière tout ce mouvement de 1946, plus particulièrement le noirisme, est-ce qu'il y avait un projet global de société ? Recherchait-on un nouveau* contrat social ?", Entretien in Cary Hector, Claude Moïse, Emile Ollivier, 1946-1976. Trente ans de pouvoir noir en Haïti. Tome premier / L'explosion de 1946. Bilan et perspectives, Québec, Collectif Paroles, 1976, p. 101. 
1804 : il date de 1801, sa forme juridique étant la constitution élaborée à l'instigation de Toussaint Louverture. Outre que cette antériorité questionne les limites de la rupture opérée par l'indépendance, elle porte l'interrogation sur la nature et la postérité du pouvoir louverturien.

L'historiographie actuelle entretient de Toussaint l'image d'un général ayant conquis et exercé un pouvoir personnel, exclusif, absolu, sans partage. Pierre Pluchon en a donné la version la plus achevée ${ }^{8}$. L'idée de la négociation d'un contrat en 1801 est inconcevable dans l'économie de cette représentation. Tout au moins, certains auteurs comme Gérard Pierre-Charles ou Claude Moïse évoquent le compromis louverturien ${ }^{9}$.

Pierre-Charles distingue trois points dans la pensée de Toussaint : "la vocation de la liberté", "le rêve de l'autonomie" et "l'utopie Noir et Blanc" "10. Il n'y aurait donc pas d'exclusion raciale de la part de Toussaint qui " devint l'expression, la plus fidèle, des lignes de forces et des pulsions de toutes natures, qui traversaient cette société en pleine ébullition revendicative et en quête d'affirmation nationale $^{11}$ ". Ces observations n'empêchent pas Pierre-Charles de dresser de Toussaint un portrait en "génie politique et militaire " de qui tout dépendait. On ne perçoit pas comment le "modèle novateur" du général s'édifierait sur un pacte social négocié. Il “ ... proclama sa propre* constitution ", un véritable instrument politique personnel.

${ }^{8}$. Pierre Pluchon, Toussaint Louverture, un révolutionnaire noir d'Ancien Régime, Paris, Fayard, 1989.

9. Yves Bénot a tenté de redéfinir ce qualificatif dans "Le compromis historique de Toussaint Louverture ", in Gérard Barthélemy et Christian Girault, La République haïtienne : état des lieux et perspectives, Paris, ADEC - Karthala, 1993, pp. 19-32. Il suggère de ne pas lire l'aventure de Toussaint “ comme s'il avait dépendu que de lui de faire ceci ou cela". Suggestion, on le verra, d'une grande valeur heuristique, en regard de l'historiographie actuelle.

${ }^{10}$. Gérard Pierre-Charles, Vision contemporaine de Toussaint Louverture, Port-auPrince, CRESFED, 1992.

11. Roger Dorsinville, Toussaint Louverture, Montréal, Port-au-Prince, CIDIHCA, 1987, pp. 189-226, cité par Gérard Pierre-Charles, op. cit., pp. 27-28. 
Claude Moïse ne dit pas le contraire, tout en effleurant la question du contrat. Il écrit en effet : "Le compromis de la politique louverturienne tend à stabiliser le régime post-colonial esclavagiste autour d'un nouveau contrat social* qui garantit la liberté générale des esclaves, élargit l'espace d'autonomie de l'Etat, reconstitue la grande exploitation avec les travailleurs agricoles fixés sur les plantations et militairement organisés, avec des fractions d'anciens colons et la couche privilégiée des nouveaux libres comme partenaires dominants". Selon Moïse, la politique louverturienne n'est pas parvenue à un nouveau contrat social, elle y tendait. Mieux, la constitution avait été commandée par "le besoin impérieux" dans lequel se trouvait Toussaint " de couronner son œuvre", le "besoin de se conforter dans la légitimité de son œuvre". Mode d'emploi qui aura une longue postérité : “ Ainsi surgit au cœur de l'Etat d'Haïti, comme plus tard au milieu des vicissitudes politiques, la question constitutionnelle en tant qu'enjeu et référence dans la reconnaissance et l'aménagement des pouvoirs. "12 Ce n'est pas le seul héritage légué à Haïti, reconnaît Moïse. Des lois et diverses dispositions constitutionnelles ont survécu au moins jusqu'à Boyer (1818-1843). Malgré tout, l'auteur de Constitutions et luttes de pouvoir en Haïti ne prend pas toute la mesure de la constitution de 1801, car il se contente de la commenter en introduction. Il n'a pu en dégager toutes les caractéristiques et implications. Ce sont là les conséquences du choix d'instituer la rupture politique de 1804 en impératif méthodologique.

Une telle option permet, au mieux, d'identifier ou de suggérer des survivances coloniales. Elle ne convient nullement à démontrer que, en dépit de la dénégation de la fraction hégémonique des élites au $\mathrm{XIX}^{\mathrm{e}}$ siècle, la structure sociale haïtienne procède de la consolidation du modèle néocolonial formalisé sous Toussaint ${ }^{13}$.

12. Claude Moïse, Constitutions et luttes de pouvoir en Haïti, Montréal, CIDHICA, 1988 , t. I, pp. 21-22.

${ }^{13}$. Il est établi que le gouvernement de Boyer a achevé la mise en place du système social haïtien. Cette structuration a consacré l'hégémonie d'une fraction des élites, les anciens libres en majorité mulâtres. Ces derniers qualifiaient le modèle louverturien de barbare et prétendaient s'en démarquer contrairement aux chefs noirs, Dessalines et Christophe, qui auraient tenté de le perdurer. Voir à ce sujet le "Précis historique " de 


\section{SAINT-DOMINGUE : LA RUPTURE DU PACTE COLONIAL VERS UN NOUVEAU CONTRAT SOCIAL}

Le pacte sur lequel reposait le fonctionnement de la colonie a été rompu en 1801. Il articulait deux niveaux de relations : les relations entre maîtres et esclaves régies par le code noir et les relations entre la colonie et la métropole définies par le système de l'exclusif.

L'édit de 1685, dit “Code noir", n'est pas une quelconque abstraction conçue dans un cabinet de Versailles sans tenir compte des réalités coloniales. Il a été élaboré par Colbert sur les mémoires (du 20.8.1682 et du 13.2.1683) de Messieurs de Blénac, Patoulet et Bégon, gouverneur-général et intendants des îles françaises de l'Amérique ${ }^{14}$. Ces mémoires consignent les propositions des colons qui avaient été sollicités en 1681. Antoine Gisler a montré le divorce entre ce code, la théorie, et les pratiques esclavagistes ${ }^{15}$. Il importe toutefois de bien noter la participation de trois entités à son élaboration : les colons, les autorités locales et le pouvoir métropolitain.

Lorsqu'en mars 1685 parut le code noir, Saint-Domingue n'était pas encore une possession pleine et entière de la France. Elle le deviendra en 1697 par le traité de Ryswick signé avec l'Espagne. Mais les français, établis d'abord à l'île de la Tortue, progressaient dans leur occupation de la grande terre. Dès le mois d'août 1685 un Edit du Roi, en forme de lettres-patentes, créa un conseil souverain et quatre sièges royaux (Goâve, Léogane, Port-de-Paix, Cap), dans la côte de l'île de

Beaubrun Ardouin dans sa Géographie de l'île d'Hä̈ti, Port-au-Prince, 1832. Pour une critique du discours historique produit par ces élites sous le gouvernement de Boyer (1818-1843), voir Victor Schœlcher, Colonies étrangères et Haïti. Résultats de l'émancipation anglaise, t. 2, Paris, Pagnerre, 1843.

14. Peytraud, Histoire de l'esclavage aux Antilles françaises avant 1789, Paris, 1897, pp. 150-166.

15. Antoine Gisler, L'esclavage aux Antilles françaises (XVII ${ }^{e}$ - XIX ${ }^{e}$ siècles). Contribution au problème de l'esclavage (1965), Paris, Karthala, 1981 (nouvelle édition revue et corrigée). 
Saint-Domingue ${ }^{16}$. Le 6 mai 1687, le conseil souverain de la côte de Saint-Domingue qui se tient au Petit-Goâve promulgue le code noir.

L'administration coloniale se mettait en place depuis 1664. A cette date fut nommé Bertrand d'Ogeron, le premier gouverneur, pour le roi, de la Tortue et Côte de Saint-Domingue. Vingt-quatre ans plus tôt, en 1640, les Français installés à la Tortue avaient appelé à leur secours Poincy, gouverneur des îles françaises de l'Amérique. Celui-ci honora leur demande et l'année suivante déclara l'île possession du roi Louis XIII. Cette prise en charge, véritable acte d'agression contre la souveraineté de l'Espagne, montre l'allure que prenaient, sur fond d'enjeux économiques, les rivalités entre pays colonisateurs. Elle supposait pour la métropole l'organisation de l'administration et de la défense de la nouvelle possession. La contrepartie avait un nom: le système de l'exclusif.

Les colons ne cesseront pas de chercher à contourner cette dernière obligation. Ils en ont fait l'objet de leur principale revendication jusqu'à la révolution. Les plus radicaux ont réclamé, pour rendre effective la liberté commerciale, l'autonomie de la colonie $^{17}$. Ils ont invoqué la spécificité du monde colonial, ont déclaré inapplicables les lois métropolitaines et ont défendu leur légitimité à légiférer. Ils ont dénoncé le despotisme ministériel et revendiqué leur droit et leur capacité à s'auto-administrer en réponse aux autorités métropolitaines qui les accusaient d'agir arbitrairement à l'égard des esclaves.

Les griefs formulés par les colons ont réussi à modifier le contenu du pacte colonial à la révolution. C'est comme une véritable entreprise de sauvetage qu'a été accueilli le rapport de Barnave ${ }^{18}$

${ }^{16}$. Michel Placide-Justin, Histoire politique et statistique de l'Ile d'Haïti, SaintDomingue, Paris, Briere Libraire, 1826, pp. 136-140.

${ }^{17}$. Sur le mouvement autonomiste des colons voir Debien Gabriel, Esprit colon et esprit d'autonomie à Saint-Domingue au XVIII ${ }^{E}$ siècle, Paris, 1954 ; Frostin Charles, Les révoltes blanches à Saint-Domingue aux XVII et XVIII siècles, Paris, Editions de l'Ecole, 1975.

${ }^{18}$. Le 3 mars est créé au sein de la Constituante le Comité colonial, dont Barnave est le rapporteur. Les travaux ont abouti à une proposition de décret présenté le 8 . Ce sentiment de sauvetage est bien exprimé par Du Morier (Dumorier), Sur les Troubles 
consacrant le principe de la spécificité des colonies. De ce rapport est sorti un décret voté le 8 mars 1790, suivi des instructions du 28 mars. Par ces dispositions l'assemblée constituante légitime les assemblées coloniales instituées par les colons. Celles-ci acquièrent la compétence de légiférer sur tout ce qui touche à l'administration interne des colonies. Les lois qu'elles auront élaborées seront soumises à la sanction des autorités métropolitaines.

Les colons, dans leur grande majorité, ont fêté l'événement. Mais les concessions étaient limitées. Aux colonies était octroyé le droit de définir leur régime intérieur, les rapports sociaux et la propriété tandis que les liens commerciaux, essentiels, entre les colonies et la métropole relevaient de la compétence de la métropole. En fait, le décret du 8 mars est le résultat d'un compromis entre les colons et les hommes du port, en France. Eux aussi, armateurs et négociants, avaient leurs griefs. Ils réclamaient la liberté du commerce, mais ne demandaient pas la même chose que les hommes de SaintDomingue, lorsque ces derniers utilisaient les mêmes mots. Pour les colons "... la liberté de transaction avec le commerce mondial assurerait aux îles, au prix le plus avantageux, les produits dont elles ont besoin ; pour les armateurs, et les négociants de Bordeaux ou du Havre, la liberté signifie la faculté pour tous, à l'exclusion de toute compagnie privilégiée, de commercer avec les îles ; étant entendu que seuls les Français doivent en profiter". Les colons mesuraient, dès septembre 1789, la nécessité de l'accord avec les villes maritimes. Celles-ci pouvaient empêcher l'irrémédiable de se produire: leurs représentants avaient investi les lieux de pouvoir. "A l'ouverture des Etats généraux, chacun a pu constater la présence dynamique, dans la révolution, des hommes du port. "19.

Mais les colons de Saint-Domingue renonçaient-ils à tout contrôle sur le commerce ? Le compromis était-il solidement scellé ?

des colonies et l'unique moyen d'assurer la tranquillité, la prospérité et la fidélité de ces dépendances de l'empire, en réfutation des deux discours de M. Brissot, des $1^{\text {er }}$ et 3 décembre 1791, Paris, Imp. de Didot Jeune, décembre 1791.

19. Jacques Thibau, Le temps de Saint-Domingue. L'esclavage et la Révolution française, Editions JC Lattès, 1989, p. 179. 
En application du décret métropolitain l'assemblée de SaintMarc adopte une déclaration en dix articles datée du 28 mai 1790, qui investit "l'assemblée générale de la partie française de SaintDomingue " du pouvoir de légiférer en tout ce qui concerne le régime intérieur de la colonie (art. 1). Les modalités des décisions d'urgence, d'exception, sont établies (art. 3, 4, 5, 7, 8). L'article premier du décret du 8 mars recommande à chaque colonie de "se conformer aux principes généraux qui lient les colonies à la métropole, et qui assurent la conservation de leurs intérêts respectifs". L'article 6 stipule : "Les mêmes assemblées coloniales énonceront leur vœu sur les modifications qui pourront être apportées au régime prohibitif du commerce entre la colonie et la métropole, pour être, sur leurs pétitions et après avoir entendu les représentants du commerce français, statué par l'assemblée nationale, ainsi qu'il appartiendra "20. L'article 6 de la déclaration de l'assemblée coloniale répond en ces termes: "Comme toutes les lois doivent être fondées sur le consentement de ceux qui doivent y obéir, la partie française de Saint-Domingue pourra proposer des règlements concernant les rapports commerciaux et autres rapports communs; et les décrets rendus à cette occasion par l'assemblée nationale n'auront force de lois dans la colonie, à moins qu'ils n'aient été consentis par l'assemblée coloniale *". Tout en étant conformes aux prescriptions du décret, les dernières précisions de cet article laissent une porte ouverte au débat sur le système de l'exclusif.

La déclaration de mai constitue la première étape vers l'élaboration d'une constitution dominguoise. L'article 10 le dit de manière précise. Il stipule: "L'assemblée générale arrête que les articles précédents, comme formant une partie de la constitution de Saint-Domingue*, seront immédiatement transmis en France, pour y recevoir la sanction du Roi et de l'assemblée nationale. Ils seront aussi transmis à tous les districts et à toutes les paroisses de la colonie, et notifiés au gouverneur-général. "21

20. "Le décret du 8 mars 1790" in Placide-Justin Michel, Histoire politique et statistique de l'Ile d'Haïti, Saint-Domingue, Paris, Briere Libraire, 1826, pp. 181-182.

21. "La déclaration du 28 mai 1790 de l'Assemblée de Saint-Marc" in PlacideJustin, op. cit., pp. 183-186. 
Un pas important venait d'être accompli. Le principe d'une constitution propre aux colonies comportait en lui-même les germes d'une éventuelle scission. Du Morier l'a probablement compris quand il a souligné que s'il faut " une organisation particulière des colonies ", " il ne peut y avoir deux constitutions dans le même empire ${ }^{22}$ ". Il y avait là une limite à ne pas franchir. Plusieurs membres de l'assemblée de Saint-Marc ne l'ont pas franchie. Ils ont refusé de signer la déclaration de mai. Toutefois le nouveau dispositif légal et administratif est en gestation. En vain ! La première constitution de Saint-Domingue ne verra pas le jour.

L'échec du compromis conclu entre les hommes du port et les colons n'est pas dû au désistement des colons " modérés". La solution envisagée niait le poids politique des esclaves et des gens de couleur. Ces groupes sociaux se jetant dans la bataille vont modifier du tout au tout les rapports de force.

Les libres de couleur, devenus numériquement et économiquement importants, pouvaient traduire en termes politiques réels leurs revendications. Ils réclamaient l'égalité civile et politique avec les Blancs en application de l'article 59 du code noir qui octroyait "aux affranchis les mêmes droits, privilèges et immunités dont jouissent les personnes nées libres". De simples et insistantes demandes (sous forme de mémoires adressés aux autorités coloniales et métropolitaines, en 1784 et 1785 par exemple) ils sont passés à l'action. Vincent Ogé a amorcé l'offensive à l'occasion du décret du 8 mars et des instructions du 28 mars 1790 qu'il a interprétés en faveur des gens de couleur ${ }^{23}$. Il a d'abord prôné l'union des propriétaires pour

22. Du Morier, op. cit. C'est l'un des points retenus dans un compte rendu de l'ouvrage de cet auteur paru dans L'Ami des Patriotes, Sur les troubles des colonies... Extrait de L'Ami des Patriotes, ${ }^{\circ}$ XVI, Imp. de Demonville, 1792, p. 6. (BNF, LK

9 191).

23. Du Morier, op. cit., a fourni, à l'époque une interprétation des dispositions légales de mars 1790 démontrant qu'elles n'ont rien concédé aux gens de couleur. Jacques Thibau, op. cit., p. 227, rapporte ce qui suit: "Avant son départ, Ogé a rencontré Barnave : l'homme du décret lui a tenu des propos imprécis tout en insistant sur l'ouverture que les Instructions apportaient aux hommes de couleur. Ogé ne lui a pas caché qu'il était déterminé à révéler "les côtés positifs du décret que l'Assemblée 
contrer une éventuelle révolte des esclaves, avant de soulever quelques hommes de sa classe pour arracher le triomphe de sa cause. Ogé passe à la roue le 26 février 1791. Le 13 mai 1791 l'assemblée nationale vote un décret constitutionnel par lequel elle reconnait qu'aucune loi portant sur les hommes de couleur ne pourrait être prise par le Corps législatif que sur la demande des assemblées coloniales. Le 15 mai elle l'amende en décrétant que les gens de couleur, nés de parents libres possèdent la qualité de citoyen et l'exercice des droits civiques qui y sont attachés. Cette nouvelle disposition est abrogée le 24 septembre. Mais quelques jours plus tôt, le 20 septembre 1791, l'assemblée coloniale, qui, le 25 août, avait bénéficié du concours des gens de couleur libres du Cap pour réprimer l'insurrection des esclaves, leur promet en échange d'accepter le décret du 15 mai. Le 23 octobre des députations de Blancs et d'affranchis des quatorze paroisses de la Province de l'Ouest de Saint-Domingue se réunissent sur l'habitation Damiens pour signer entre eux un traité de paix, connu sous le nom de Concordat de Damiens. ${ }^{24}$ Le 4 avril 1792 l'assemblée législative prend un décret reconnaissant aux libres de couleur l'égalité civile et politique avec les Blancs.

Ogé a payé de sa vie, mais l'union des propriétaires est devenue une condition nécessaire à la sauvegarde de la colonie. Cette stratégie se révélera incapable de contenir la révolte des esclaves déclenchée dans la nuit du 22 au 23 août 1791.

Les 9 et 12 décembre des émissaires des révoltés se présentent à l'assemblée coloniale en vue de négociation. Aucune réponse. Le 15, l'assemblée les informe que " fondée sur la loi et par la loi, (elle) ne peut correspondre avec des gens armés contre la loi, contre toutes les lois. " Elle leur précise qu'elle “pourrait faire grâce à des coupables repentants et rentrés dans le devoir. Elle ne demanderait pas mieux que d'être à même de reconnaître ceux qui ont été entraînés contre leur

nationale avait rendu en faveur des hommes de couleur". Barnave ne l'a pas contredit".

24. Ce document est reproduit dans Chemins Critiques, Revue HaïtianoCaraïbéenne, Vol. 2, n 3, mai 1992, pp. 261-281. 
volonté. Elle sait toujours mesurer sa bonté et sa justice". Puis elle ordonne aux représentants des nègres de se retirer ${ }^{25}$.

Cette attitude ne sera pas celle adoptée par les commissaires, Roume, Mirbeck et Saint-Léger, envoyés par la métropole (ils sont arrivés le 28 novembre 1791) pour rétablir l'ordre dans la colonie. Ils ont adressé une lettre aux chefs des insurgés les invitant à parlementer avec eux. Le 21 décembre Mirbeck et Jean-François, chef des rebelles, se sont rencontrés. Le commissaire dira que Jean-François avait promis de faire rentrer les révoltés dans le devoir; le général Pamphile de Lacroix rapporte que celui-ci avait réclamé quatre cent libertés. En tout cas, les négociations n'ont pas été fructueuses. Dans une lettre du 15 février 1792, les commissaires ont mis cet échec sur le compte d'hommes criminels qui auraient armé les nègres. On les aurait persuadés qu'ils voulaient les désarmer pour les exterminer. De son côté l'assemblée ne voulait rien accorder.

Le 21 septembre 1792, Sonthonax débarque à Saint-Domingue accompagné de Polvérel et Ailhaud. Cette deuxième commission civile, envoyée par la Convention montagnarde, est chargée de ramener l'ordre dans la colonie en faisant exécuter le décret du 4 avril. Le développement des luttes politiques portera Sonthonax à outrepasser les limites de cette mission.

Du 20 au 23 juin 1793, le nouveau gouverneur, Galbaud, propriétaire d'habitation dans la plaine de Léogâne, tente un coup de force contre les commissaires. Ces derniers matent la rébellion en recourant à l'appui des esclaves insurgés des mornes environnants. Galbaud s'enfuit, bientôt suivi par dix mille colons environ. Le poids de la force des esclaves mise dans la balance a fait basculer les choses. La crainte d'un débordement de cette force, la nécessité de la contrôler pour mieux l'utiliser ont déterminé la solution de la liberté générale ${ }^{26}$.

25. Lettre du marquis de Rouvray citée par Jacques Thibau, op. cit., p. 323.

26. Les commissaires auraient tout fait pour se rallier Jean François et Biassou : envoi d'émissaire, correspondance secrète, tentative d'attaques. Mais en vain ! Les succès des troupes de ces derniers, opérant à partir de la zone espagnole, inquiétaient alors que Sonthonax disposait de peu de soldats dans une ville, le Cap, entourée d'un nombre considérable de noirs... Selon Michel Placide-Justin, op. cit., pp. 257-262. 
L'opportunité de cette solution a été discutée. Et c'est à l'issue de délibérations que la commune du Cap a voté, le 24 août, la liberté générale dans la province du Nord. Jacques Garnier, commissaire du pouvoir exécutif au siège du tribunal du Cap en a témoigné : "Ils (les esclaves) étaient devenus les défenseurs du sol de la liberté : il fallait enfin les rendre à leurs droits naturels. La commune du Cap français s'assembla le 24 août 1793. Les hommes libres qui la formaient, le bonnet de la liberté au milieu d'eux, eurent l'honneur de voter les premiers à l'unanimité pour la liberté générale des Africains et descendants qui se trouvaient sur le sol de la province du Nord. L'acte fut dressé et porté pour sa sanction provisoire au milieu des cris de Vive la République française, répétés par quinze mille âmes au commissaire Sonthonax, qui reçut l'adresse de la commune comme l'expression de la justice et de l'humanité, qui appelaient à grands cris depuis deux siècles l'abolition de l'esclavage.

Florence Gauthier qui rapporte ces propos commente à juste titre: "Sonthonax ne prit pas de son propre chef la décision, on le voit, il répondit au mouvement de ces nouveaux citoyens qui combattirent de juin à août pour la liberté générale "28. Le commissaire publie la

27. A.N. DXXV 82, dossier 804, Mémoire de J. Garnier, commissaire du pouvoir exécutif au siège et au tribunal du Cap, 7 novembre 1794, 34 p.; voir aussi l'intervention de Dufay, Archives parlementaires, tome 84, "Convention, séance du 16 pluviôse an II (4 février 1794) ”, p. 277. Cités par Florence Gauthier, "Le rôle de la députation de Saint-Domingue dans l'abolition de l'esclavage", in Les Abolitions de l'esclavage de L.F. Sonthonax à V. Schœlcher, 1793, 1794, 1848, textes réunis et présentés par Marcel Dorigny, Presses Universitaires de Vincennes et Editions Unesco, 1995, p. 203.

27. Pluchon, op. cit., p. 89.

28. Tout le contraire de la lecture de Pierre Pluchon. "Désorienté par les bouleversements locaux et internationaux, par l'isolement et la fragilité de son pouvoir le représentant de la République joue son va-tout. Le 29 août 1793, sans consulter ses collègues, poussant à son terme la politique libératrice qu'il a amorcée, il abolit l'esclavage, proclame la liberté générale"; ou bien ce passage dans lequel l'auteur parle d'une "décision brutale qui serait longtemps préparée..., Toussaint Louverture, Paris, Fayard, 1989, p. 81. Cette version de l'initiative personnelle, exclusive de Sonthonax a été repris de sa propre relation des faits. Dans son compte rendu justificatif envoyé aux "Sociétés des Amis de la Liberté et de l'Egalité en France" il écrit, après avoir rappelé les circonstances de la décision: "Pressé par tant de 
proclamation le 29 août. Il élargit la liberté générale à l'ensemble de Saint-Domingue en chargeant Polvérel de la proclamer dans les autres parties de la colonie. Le 21 septembre l'esclavage est aboli dans l'ouest et le sud. Il ne restait qu'à obtenir l'approbation de la métropole. Une députation, élue le 23 septembre, selon le principe de l'égalité de l'épiderme (trois Noirs, trois Blancs et trois Métis), est chargée de cette mission $^{29}$. Elle est admise à la Convention le 15 pluviôse an II (3 février 1794). Dès ce moment, explique Florence Gauthier, la République reconnaît la liberté générale des noirs devenus citoyens ainsi que la décision de Sonthonax et de Polvérel. Ainsi, lorsque, le lendemain, l'un des députés, Dufay, expose longuement le mandat de

circonstances, et au moment de voir passer dans des mains ennemies la propriété de Saint-Domingue, je n'ai pas hésité* de proclamer la liberté générale dans la province du Nord...". Cette adresse est reproduite dans Joachim Benoît, Les racines du sousdéveloppement en Haïti, Port-au-Prince, Imprimerie H. Deschamps, 1979, pp. 29-31. Selon Victor Schœlcher (1843 !) la décision de Sonthonax " ne fut qu'un des accidents du terrible drame qui se jouait sur cette terre d'esclavage ". Il précise et souligne : "il y fut contraint et forcé". Il ajoute, se référant à Malenfant, que "plusieurs blancs du Cap, et parmi eux M. Artau, propriétaire de mille esclaves, la jugèrent nécessaire et engagèrent Sonthonax à la prendre ". Il poursuit plus loin en rappelant que Polvérel, son collègue le désapprouva publiquement. "Mais quand Polvérel vit la guerre civile ranimée par le débarquement des Anglais, il jugea bien qu'il serait impossible de contenir plus longtemps les nègres, et il engagea le petit nombre de propriétaires restés fidèles à concourir à un acte qui devait les sauver. Ceux-ci, en conséquence, signèrent de leurs propres mains la liberté de leurs esclaves. Malenfant, qui rapporte ces détails, dit que la liste des signataires fut imprimée, et ajoute sans commentaire : "Je suis le seul blanc qui ait refusé de signer" ", op. cit., p. 113 et p. 114.

29. Cette forme de représentation n'est pas une invention de Sonthonax. Elle est envisagée dès les tentatives de résolution du problème des "libres de couleur", cette catégorie étant constituée de noirs et de mulâtres. Carteaux Félix, Histoire des désastres de Saint-Domingue. Soirées Bermudiennes ou entretiens sur les événemens qui ont opéré la ruine de la partie française de l'île St-Domingue, Bordeaux, PelletierLawalle, 1802, p. 165. Julien Raimond, dans son mémoire présenté au mois de juin 1793 au comité des colonies à la Convention (Mémoire sur les causes des troubles et des désastres de la colonie de Saint-Domingue, Paris, Imp. du Cercle social, 1793, p.) considère que la question de couleur est la cause fondamentale des malheurs de la colonie. Une de ses propositions: la constitution de compagnies soldées et non soldées. Pour cela “ ...il sera choisi parmi les individus de chaque couleur, des citoyens ayant des propriétés qui répondront respectivement des hommes de leur couleur qu'ils feront entrer dans ces compagnies...", p. 63. 
la députation, il ne s'agit point d'un plaidoyer pour l'abolition déjà acquise. "Il s'agit alors d'autre chose. Dufay était mandaté, avec Belley et Mills, pour proposer un contrat d'association entre le nouveau peuple de Saint-Domingue, qui avait lui-même conquis la liberté, et le peuple de France qui menait lui aussi sa lutte pour la liberté. Dufay proposait à la République française une politique commune de guerre contre les colons esclavagistes français et leurs alliés anglais et espagnols". [...] "La Convention accepta cette offre, puis décida d'élargir la liberté conquise à Saint-Domingue en liberté générale dans toutes les colonies françaises. Elle vota à l'unanimité une Déclaration d'abolition de l'esclavage dans les colonies, base du nouveau contrat d'association entre ces peuples, suivie d'un décret d'application qui élargissait la citoyenneté aux intéressés..." ${ }^{\text {30 }}$ le 16 pluviôse an II (4 février 1794).

Le lobby esclavagiste riposta contre la députation de SaintDomingue et continua son combat jusqu'au rétablissement de l'esclavage en 1802. A Saint-Domingue, dès le 3 septembre, les colons esclavagistes avaient signé un traité avec les Anglais leur livrant la colonie. L'occupation anglaise, s'accompagnant du rétablissement de l'esclavage, s'étendait à une grande partie de la colonie.

C'est dans la lutte pour la reconquête de Saint-Domingue que Toussaint allait se distinguer et prendre une réelle ascendance. Abandonnant le camp espagnol, il combattit les Anglais et rendit la colonie à la France. Il s'employa à atteindre l'ultime échelon du pouvoir et s'y maintenir, en se donnant comme objectif primordial la consolidation des acquis de la liberté. Il se débarrassa des représentants de la métropole: Sonthonax en août 1797, Hédouville en 1798 ; contrôla Roume. Il neutralisa les anciens libres ; à l'issue de la guerre du Sud, en juillet 1800, leur chef Rigaud, vaincu, est contraint de quitter la colonie. Le 26 janvier 1801 le général Toussaint entre dans Santo Domingo où il proclame la liberté générale. Il réunifie l'île après avoir signé courant 1798-1799 des traités militaires et commerciaux

${ }^{30}$. Florence Gauthier, op. cit., p. 204-205. Une toute autre lecture est proposée par Pluchon: Sonthonax "a imposé la suppression de l'esclavage à une Convention hésitante et contrariée ", écrit-il, op. cit., p. 546. 
avec l'Angleterre et les Etats-Unis, rompant ainsi le système de l'exclusif.

Saint-Domingue est de fait autonome, au moment où la métropole entend revenir sur le principe acquis de l'autonomie des colonies. La Constitution de l'an VIII (13 décembre 1799) établissant le Consulat maintient que "Le régime des colonies françaises est déterminé par des lois spéciales ", mais elle leur enlève le droit de les préparer elles-mêmes pour les soumettre à la sanction de la métropole. "Un des premiers actes de la législature sera la rédaction de lois destinées à vous régir. Loin qu'elles soient pour vous un sujet d'alarme, vous y reconnaîtrez la sagesse et la profondeur des vues qui animent les législateurs de la France." C'est ce qui est annoncé aux autorités de Saint-Domingue dans une proclamation officielle du Premier Consul, confiée à des émissaires nommés en vue de la réorganisation de l'administration de la colonie. Le pouvoir métropolitain continue à mal évaluer l'évolution des rapports de force à Saint-Domingue. Les délégués du Consulat, le colonel Vincent, Julien Raimond, le général Michel, munis de papiers qui datent du 25 décembre 1799, quittent la France au mois de mai 1800, débarquent à Santo Domingo au mois de juin, puis regagnent la partie occidentale de l'île. La nouvelle Constitution française et la proclamation du Premier Consul n'ont pas été publiées. Toussaint achevait de consolider sa position dans le but évident d'éviter un retour à l'ordre ancien.

Le 4 février 1801, de Santo Domingo, il convoque une assemblée, dénommée "assemblée centrale de Saint-Domingue", chargée de la rédaction d'une constitution. Les travaux se déroulent à Port-au-Prince du 22 mars 1801 au 9 mai 1801. Le 3 juillet Toussaint approuve la charte et la proclame publiquement le 8 du même mois. Par une lettre du 16 juillet confiée au colonel Vincent, Français, directeur des fortifications, Toussaint, désormais gouverneur, demande la sanction de Bonaparte. Mais en vertu de son dernier article (art. 77), la nouvelle charte est déjà entrée en application. Du 6 juillet au 6 août, 
le gouverneur en accord avec l'assemblée centrale, a promulgué un ensemble de lois mettant ainsi en place les nouvelles structures ${ }^{31}$.

Tout cela se déployait sur fond d'incertitude et, en fin de compte, ne dura que quelques mois. En guise de sanction officielle de sa constitution, Toussaint reçut une armée expéditionnaire. Le général Leclerc, commandant des troupes françaises, arriva devant le CapFrançais le 29 janvier 1802. C'est la guerre. Le 7 juin Toussaint est arrêté, puis déporté. La brièveté de l'expérience louverturienne n'enlève rien à sa valeur fondatrice. Elle a la valeur de ces " séquences assez brèves", ces moments assez rares mais déterminants qui constituent, selon Alain Badiou, "l'événement politique". ${ }^{32}$ Les données et les configurations sont puissamment déplacées; de "nouvelles prescriptions " commandées par de " nouvelles virtualités" sont rendues possibles. Le contrat colonial a été rompu et rien ne sera comme avant. Un nouveau contrat a été négocié.

La négociation s'est déroulée dans un contexte spécifique, celui d'un renversement des rapports de force. Ce préalable est à prendre en compte pour caractériser les acteurs impliqués et leur représentativité. L'impératif a été de définir un nouveau modèle social. Deux données inséparables y seront déterminantes: le choix de la structure économique et l'articulation du rapport liberté/travail. La solution apportée ne sera pas sans fondements théoriques et idéologiques repérables.

${ }^{31}$. Loi du 6 juillet divisant l'île en 6 départements ; loi du 15 juillet sur le culte catholique; loi du 23 juillet organisant la justice ; loi du 25 juillet sur les officiers ministériels; loi du 30 juillet sur la garde nationale non soldée ; loi du $1^{\text {er }}$ août sur les dettes particulières ; loi du 3 août sur l'administration des Finances ; loi du 6 août sur les émigrés et les délits; le règlement des cultures avait été prise le 12 octobre 1800 , renforcé par une proclamation du 20 novembre 1801.

32. Alain Badiou, “Nous pouvons redéployer la philosophie”, Entretien avec Roger-Pol Droit, Le Monde, 31 août 1993, recueilli dans "Les grands entretiens du Monde", t. 2, mai 1994, p. 30. 


\section{LE CONTRAT DE 1801 \\ FORMALISATION D'UN NOUVEAU MODELE SOCIAL. NOUVEAU JEU DES FORCES.}

\section{a. - Conditions de négociation et représentativité des acteurs}

De Pamphile de Lacroix (1819) à Claude Moïse $(1988)^{33}$ en passant par Michel Placide-Justin $(1826)^{34}$ et Louis Joseph Janvier $(1886)^{35}$, on s'appuie sur une citation prêtée ${ }^{36}$ à Toussaint pour certifier que la constitution de 1801 n'a été qu'un simple instrument de consécration de son pouvoir personnel. Le général aurait déclaré : " J'ai pris mon vol dans la région des aigles. Il faut que je sois prudent en regagnant la terre ; je ne puis plus être placé que sur un rocher, et ce rocher doit être l'instrument constitutionnel qui me garantira le pouvoir tant que je serai parmi les hommes ".37

${ }^{33}$. Claude Moïse, op. cit., p.22.

${ }^{34}$. Michel Placide-Justin, op. cit., p. 340.

${ }^{35}$. Louis Joseph Janvier, Les constitutions d'Hä̈ti (1801-1885), Paris, C. Marpon et E. Flammarion, 1886, p. 2.

36. Le Général Pamphile de Lacroix qui a été, peut-être, le premier à le rapporter n'en donne pas les références exactes. "Une locution, qu'il avait adoptée avant le retour du commissaire Raimond [1800 ] ... ”, écrit-il. Général Pamphile de Lacroix, La révolution de Haïti (1819) édition présentée et annotée par Pierre Pluchon, Paris, Karthala, 1995, p. 259.

${ }^{37}$. Les commentateurs, considérant sa chute, en concluront : de fait il est allé trop loin. Il en serait conscient et accepterait de se sacrifier pour le bonheur des autres. Cette lecture qui a fait fortune de l'histoire au théâtre reprend une image récurrente de la légende des " grands hommes ". Edouard Glissant écrira : "Dans les Jacobins noirs, C.L.R. James soutient que Toussaint-Louverture, premier artisan de l'indépendance de Saint-Domingue, ne garda pas le contact avec la révolution populaire ; qu'il se vit ainsi à partir d'un apogée de puissance, abandonné de ceux qu'il avait libérés de la servitude. Aimé Césaire (dans son Toussaint-Louverture) consent à la thèse de James et la complète : supputant que Toussaint, en effet conscient d'être dépassé par la situation, incapable de faire le saut radical de l'indépendance, convaincu peut-être que sa présence rendait impossible la réconciliation entre les Nègres et les Mulâtres, et, aussi, incliné à une conception tragique de son destin révolutionnaire, délibérément ou non se sacrifie à la cause commune et trouve dans un tel sacrifice l'achèvement 
Cette "locution, [...] adoptée avant le retour du commissaire Raimond [1800], démontre jusqu'à l'évidence que le plan d'une constitution coloniale était depuis longtemps dans la tête du chef des Noirs", confie Pamphile de Lacroix. Depuis la déclaration de l'assemblée de Saint-Marc du 28 mai 1790 la rédaction d'une constitution dominguoise était en attente. Toussaint pouvait fort bien inscrire dans ses objectifs sa matérialisation. Mais cette constitution a été préalablement préparée, selon certains historiens. Pluchon, qui le réaffirme, insiste sur un autre point: les constituants étaient des " créatures" de Toussaint. "L'Assemblée centrale de Saint-Domingue est constituée de créatures zélées, écrit-il. Comme le créole [Toussaint] le dira à Caffarelli, les dix députés qui reçurent l'onction populaire, étaient "en grande partie à sa dévotion"". Qui sont ces hommes ? "Parmi eux, pas un Noir! Mais trois mulâtres, Raimond, Viart et Lacour, trois Blancs français, Borgella, Collet, Nogerée et quatre Espagnols, Roxas, Muñoz, Mancebo et Morillas (ce dernier mourra sitôt après sa désignation). [...] Borgella, planteur aisé, ancien avocat au Conseil supérieur du Port-au-Prince, est connu pour ses opinions autonomistes. Il est nommé président. Ses deux compatriotes, Nogérée (sic), qui en 1793 avait appelé les Anglais à Jérémie, et Collet, autre sudiste, serviront d'acolytes. Raimond, ancien défenseur des gens de couleurs au début de la Révolution, puis commissaire du Directoire dans l'île, est favorable aussi à un système d'autonomie. Viart, plus neutre est nommé secrétaire de l'Assemblée. Les Espagnols, qui participeront aux travaux constituants, siègent pour la forme". Pluchon poursuit: "Personne ne se fait d'illusion sur l'issue des débats qui vont s'engager. Dès le 6 mars 1801, Périès informe le ministre de la Marine que les députés présenteront une constitution déjà fabriquée, fondée sur le prétendu voeu du peuple de créer Toussaint, chef suprême de la colonie. Effectivement, confirme

politique de son action. Glissant ajoute "Ces vues d'ensemble me furent profitables." Monsieur Toussaint (version scénique), Paris, Seuil, 1986, Préface à la première édition (1961), p. 7. Claude Moïse reprend le même langage : "Ainsi Toussaint est allé très loin, mais trop loin de sa base de masse, et beaucoup trop loin du pouvoir métropolitain dont il s'est débarrassé des agents ", op. cit., p. 22. 
Pluchon, Borgella et Louverture s'étaient accordés sur la rédaction d'un texte, préalablement à la réunion des représentants ". ${ }^{38}$

L'auteur ne fournit aucune précision concernant cette dernière affirmation ; il ne renvoie à aucun document qui en attesterait. De plus, s'accorder sur la rédaction d'un texte signifie-t-il s'accorder sur un texte préalablement fabriqué? D'ailleurs, on ne comprend pas pourquoi les constituants ne devraient pas être préalablement d'accord sur le principe. Cela autorise-t-il à considérer les membres de l'assemblée centrale comme de simples créatures de Toussaint? Borgella et Raimond se sont-ils ralliés aux positions de Toussaint pour agir dans le sens de ses intérêts ou de leurs propres intérêts et ceux de leurs groupes sociaux ?

L'autonomie de Saint-Domingue s'est concrétisée, mais sous l'impulsion d'une autre force politique que celle qui l'avait conçue en tant que projet politique. Résultat : le système de l'exclusif est rompu, l'autonomie administrative traduite dans les faits, la liberté générale des esclaves consacrée, l'égalité juridique entre les citoyens proclamée. Les revendications des trois catégories sociales de la colonie sont satisfaites, selon une configuration qui annule les tendances de chacune d'elles à l'exclusivisme. Une situation nouvelle s'est créée. Elle a été prise en charge par une avant-garde, ceux qui, pénétrés des acquis historiques, ont cru possible et nécessaire un nouvel ordre des choses. Cette avant-garde, comme toujours dans ces cas, n'a pas reçu le suffrage de tout le monde. Mais c'est au niveau de sa composition qu'il faut poser le problème de la représentation des groupes sociaux, de la représentativité des tendances et des acteurs qui en sont les voix. Borgella, Raimond et Toussaint sont trois figures de l'avant-garde de $1801^{39}$. Le premier représente l'intérêt des colons, les autonomistes en particulier, le second celui des gens de couleur, le troisième, ancien libre, s'est porté garant de la liberté générale des esclaves.

38 . Pierre Pluchon, Toussaint Louverture, Paris, Fayard, 1989, p. 373.

${ }^{39}$. On excepte les représentants de la partie espagnole dont la présence ne semble pas avoir été déterminante. En tout cas ils ne sont pas pertinents pour la suite de l'analyse. 
La révolution sociale en cours à Saint-Domingue ne se mesure pas uniquement à l'aune de la liberté générale. Les élites domingoises se reconstituent, dessinant la première configuration de la classe dominante haïtienne. Celle-ci n'a pas été modifiée par la guerre de l'indépendance. Elle en est plutôt sortie légitimée, pour avoir été aux commandes des opérations. A l'issue des affrontements, recueillant le pouvoir, elle a entrepris de se raffermir sur la base du modèle social formalisé en 1801. Cette postérité du modèle confère un relief particulier au rapport de Raimond et de Toussaint. Leurs rôles respectifs ont été décisifs tant dans l'élaboration du nouveau projet de société que dans la composition des nouvelles élites qui en achèveront la mise en œuvre.

Qui est Julien Raimond et quel a été son rôle ?

Julien Raimond devenu constituant était revenu à SaintDomingue en 1800 en qualité d'émissaire du Consulat. Avant cette ultime mission, il fut membre de la troisième commission civile, envoyée en 1796 par le Directoire et dirigée par Sonthonax. Seul représentant de la métropole au départ de celui-ci, il regagna la France en 1798 avec Hédouville. Entre 1796 et 1801 Raimond incarne la participation des hommes de couleur à l'administration de la colonie, après avoir milité pendant longtemps pour l'obtention de ce droit. Ce grand propriétaire de caféiers dans la partie sud de la colonie, avait engagé la défense des gens de couleurs une décennie plus tôt. En 1784, il remit au gouverneur Bellecombe un mémoire contre le préjugé de couleur et en faveur de l'égalité des droits avec les Blancs. Il renouvela sa démarche l'année suivante auprès du maréchal de Castries, secrétaire d'Etat de la Marine. Au mois de décembre 1789, il adhéra à la Société des Amis des Noirs. Le 15 mars 1789, il réclama, avec son frère, François, la représentation des gens de couleur libres aux Etats Généraux. Le 26 août il présenta la revendication des hommes de couleur devant le club Massiac, société des propriétaires de SaintDomingue résidant en France. Avec Vincent Ogé, il s'inscrivit à la Société des colons américains, dénomination à partir du 12 septembre de l'Assemblée des gens de couleur des îles et des colonies françaises fondée le 29 août 1789. Le 13 mai 1791, il prit la parole au club des Jacobins, introduit par Robespierre ; le 15 mai, il fut admis à la barre 
de l'assemblée nationale pour y défendre les droits des gens de couleur. En 1793, il fit partie d'une commission de consultation auprès de la Convention. Dans son premier mémoire au Roi, il écrivit : "La classe des personnes de couleur est sans contredit le plus sûr appui des Blancs contre la rébellion des esclaves. C'est ainsi qu'en a jugé par expérience un administrateur philosophe qui a consigné ces faits dans une note très longue de l'Encyclopédie, à l'article mulâtre. Les hommes de couleur ont les mêmes intérêts que les Blancs dans la colonie, celui de la conservation de leurs biens et celui de maintenir les esclaves dans les bornes que leur état prescrit." Il poursuivit: "Personne n'est plus agile pour gravir les mornes et ramener les nègres marrons que nos gens de couleur, qui font seuls la sûreté de la colonie contre la révolte et le marronnage. "40

Toussaint n'ignorait pas ce discours esclavagiste, comme il le laissait comprendre en parlant de Vincent Ogé qui tenait les mêmes propos $^{41}$. Il connaissait Raimond. On a même insinué que celui-ci l'aurait incité à se saisir du pouvoir comme venait de le faire le général Bonaparte, en France par la révolution du 18 Brumaire. Il en faisait peu de $\operatorname{cas}^{42}$. Quoi qu'il en soit les deux hommes étaient devenus proches. Raimond s'était déjà adapté aux nouvelles donnes de la conjoncture. En 1793, il prônait à la Convention l'amélioration du sort des esclaves pour sauvegarder la colonie ${ }^{43}$ avant de devenir, après la liberté générale, commissaire du pouvoir métropolitain.

${ }^{40}$. Premier mémoire de Julien Raimond au Roi, $\mathrm{n}^{\circ}$ 77, cité par Jacques Thibau, op. cit., p. 229.

${ }^{41}$. A propos d'une lettre de Villate, Toussaint confie à Laveaux, le 21 avril 1796 : "Je me suis aperçu par le style et le passage de la mort des prétendus martyrs de la liberté générale : Ogé et Chavannes, que l'auteur de ces deux lettres est un homme de couleur. Il impose impunément quand il dit que ces deux chefs moururent pour la liberté. J'ai des preuves par devers moi qui m'assurent le contraire. Au premier moment, je vous les enverrai, ainsi qu'à Pierre Michel ". Cité par Pierre Pluchon, op. cit., p.131.

${ }^{42}$. Général Pamphile de Lacroix, op. cit., p. 259.

43. Son rapport est indiqué en note 29. Voir aussi ses Réflexions sur les véritables causes des troubles et des désastres de nos colonies, notamment sur ceux de SaintDomingue avec les moyens à employer pour préserver cette colonie de la ruine totale ; adressée à la Convention nationale, Paris, 1793. 
Le 23 janvier 1796, le Directoire demande l'envoi d'une troisième commission civile à Saint-Domingue. Celle-ci y est arrivée le 11mai 1796, composée de Roume, Sonthonax, Leblanc, Giraud et Raimond. Roume est affecté à la partie orientale. Sonthonax qui a l'œil sur tout, est officiellement chargé des affaires militaires. Leblanc est responsable du commerce, Giraud des finances et Raimond s'occupe des cultures. L'équipe se retrouve dans une colonie en pleine transformation. Ses mesures vont en accélérer le processus.

La colonie, un moment coupée de la métropole à cause de la guerre en Europe, devait subvenir à ses propres besoins. Cette situation encouragea les cultures vivrières au détriment des denrées d'exportation. De plus beaucoup de propriétaires avaient émigré depuis les troubles, laissant leurs habitations inexploitées. Des mesures s'imposaient pour redresser l'économie de plantation et combler les déficits de l'administration coloniale. La principale solution adoptée par Giraud et Raimond a été l'affermage des biens séquestrés. Cette politique sera poursuivie jusqu'à ce que Toussaint occupe le sommet du pouvoir, ascension devenue effective à partir de l'expulsion de l'agent Hédouville en 1798. Raimond y sera associé, car, chargé des cultures, à son retour à Saint-Domingue en 1800, il s'occupera de l'administration des domaines sous Toussaint.

La brèche ouverte par la politique de location $^{44}$ des terres profitait dans un premier temps aux anciens propriétaires restés sur place dont des anciens libres. "C'est bien Rigaud qui dans le Sud conduit efficacement l'appropriation des biens vacants ${ }^{45}$. Sous Toussaint elle a permis à des nouveaux libres d'accéder à la propriétét ${ }^{46}$. Ce sont les officiers de l'armée qui en ont bénéficié, dans

${ }^{44}$. Qui n'excluait pas l'accaparement de force de biens vacants. Ce qui d'ailleurs faisait l'objet de litiges entre les représentants du pouvoir central et certains responsables militaires. Voir Vertus Saint-Louis, "Régime militaire et règlement de culture ", in Chemins Critiques, vol. 3, $\mathrm{n}^{\text {os }} 1$-2 1993, p. 183-227.

45 . Ibid, p. 198.

46. "Les ordres du gouvernement y parvenaient difficilement [dans le Sud]. Les plus considérables propriétés et les plus épargnées par les troubles furent affermées à vils prix aux chefs militaires ; de la jouissance naquit le droit de propriété*. On soutint ensuite sa possession du droit de la force", Leborgne du Borgne, Nouveau système 
un cas comme dans l'autre. La classe des possédants de la future Haïti est désormais constituée de deux branches qui se battront pour le partage des biens et l'hégémonie politique ${ }^{47}$. Elle restera marquée par les circonstances de son avènement. Devenue dominante grâce au transfert de la propriété coloniale, elle s'assumera avant tout en tant que classe de substitution. Dans un contexte différent, certes, l'esclavage ayant été aboli, mais la structure économique est maintenue. Ce nouvel ordre social est défini par la constitution de 1801.

\section{b. - Le jeu des forces et le modèle social dans le texte constitutionnel}

L'interprétation de la constitution de 1801 semble être généralement surdéterminée par son titre VIII (articles 27-41) traitant du Gouvernement. Par ces quinze articles, en effet, un pouvoir étendu est confié à Toussaint nommé gouverneur général à vie. Dans quelle mesure faut-il n'y voir que le besoin d'un pouvoir sans partage ? $\mathrm{Ne}$ faut-il pas au moins se poser la question de la nécessité d'un tel pouvoir ? Quoi qu'il en soit les constituants ont conçu des mécanismes de régulation. Conscients du caractère exceptionnel de la conjoncture, ils misent avant tout sur le temps. “... Tous les deux ans, les Assemblées centrales suivantes pourront opérer les changements que le temps et l'expérience rendront nécessaires ". Cette flexibilité, rappelée dans le discours préliminaire, laisse ouverte la possibilité d'un meilleur équilibre des pouvoirs. D'autant que les élections pour le renouvellement de l'assemblée (art. 22 et 23), tous les deux ans, ne concerneront qu'une catégorie sociale bien ciblée, les propriétaires. Mais déjà l'assemblée s'octroie une marge de manoeuvre par rapport

pour la colonisation de Saint-Domingue, 1817, Paris, pp. 126-136, cité par Vertus Saint-Louis, op. cit., p.198.

47. Les deux étant évidemment liés, comme le prouve l'assassinat de Dessalines, premier épisode de cette lutte après l'indépendance, ainsi que la crise de sa succession. La question du partage se manifestera à travers les politiques de distribution des terres entreprises par les gouvernements successifs. 
au gouverneur (art. 19-26). S'il revient à ce dernier de proposer les lois, c'est elle qui les rend (art. 20), mieux elle " vote l'adoption ou le rejet des lois qui lui sont proposées par le gouverneur; elle exprime son vote sur les règlements faits et sur l'application des lois déjà faites, sur les abus à corriger, sur les améliorations à entreprendre dans toutes les parties du service de la colonie" (art. 24). Elle se prononce aussi sur les recettes et les dépenses, la perception des impôts (art. 26).

Les ressorts de ces mécanismes se comprennent davantage à la lecture d'un autre passage du discours préliminaire. La constitution y est présentée comme " un code de lois auquel viendront se lier toutes les affections, se réunir tous les intérêts". Il y est précisé que "Le peu de membres dont il [Toussaint ] a formé cette Assemblée annonce qu'il a voulu éloigner de ses discussions les passions et les tumultes ; mais en même temps, il a voulu qu'elle fût environnée de tous les hommes instruits* , afin qu'un ouvrage d'un si grand intérêt* fût, pour ainsi dire, celui de la colonie entière*". Les constituants soulignent que "Si l'Assemblée centrale n'a pas complètement rempli les voeux de ses commettants, si elle n'a pas atteint le but que se proposait le général en chef*, elle aura fait au moins ce que les circonstances lui permettaient. Elle n'a pu proposer à la fois tous les changements qu'on pouvait désirer*. "Une fois de plus ils s'en remettent à l'œuvre du temps. "La colonie ne peut parvenir à la plus grande prospérité qu'avec le temps, et par degré. Le bien, pour être durable, ne peut s'opérer que lentement; il faut, à cet égard, imiter la nature qui ne fait rien avec précipitation, mais qui mûrit peu à peu ses productions bienfaisantes*. Heureux si cette première tentative peut contribuer à améliorer le sort de ses concitoyens*, et à lui mériter leur estime et leur indulgence, aussi bien que les témoignages de satisfaction de la France, quand bien même elle n'aurait pas atteint une certaine perfection".

Comment ne pas tenir compte de ce que dit de manière explicite cette citation ? Peut-on la considérer comme du simple verbiage ?

Pluchon relève à juste titre qu'aucun Noir ne figure parmi les constituants. Mais pour lui, la constitution n'exprime en rien les intérêts des Blancs et Mulâtres qui l'ont rédigée. Il a cependant bien rappelé que, prenant la parole au nom de ses pairs pour exposer la 
genèse et les finalités de la constitution, le président de l'assemblée, le colon planteur Borgella, ne s'est adressé qu' "aux colons français" et "aux braves soldats"

Dans un contexte où les conflits raciaux sont encore vifs, l'absence de Noirs à l'assemblée centrale (quand il y avait trois Noirs, trois Blancs et trois "Métis" à la Convention en 1793 !) paraît autrement plus importante quand les constituants se proclament des hommes instruits, en tant que tels seuls aptes à représenter la colonie entière. Il s'agit là d'une revendication de l'exercice du pouvoir par une catégorie sociale. Le savoir légitime le pouvoir en conférant une représentativité exclusive et englobante (la colonie entière). Cette opération idéologique est importante à plus d'un titre. Au moment où la liberté générale des esclaves fait vaciller la configuration sociale coloniale articulée sur la hiérarchie des races, cet énoncé reconduit le même dispositif en doublant la couleur du savoir. Cette construction idéologique promettait d'être efficace dans un contexte où ceux qui avaient accès à ce savoir dont il est question provenaient de la catégorie des propriétaires, ici précisément les anciens propriétaires où se recrutaient peu de Noirs. De plus le système qui se mettait en place n'envisageait pas d'étendre au plus grand nombre possible ce savoir qui confère le pouvoir ${ }^{49}$. On a là peut-être les prémisses d'une ligne idéologique qui sera synthétisée vers la fin du $\mathrm{XIX}^{\mathrm{e}}$ siècle dans la formule le pouvoir aux plus capables. La réplique en sera le pouvoir au plus grand nombre, à l'œuvre déjà, selon Roger Dorsinville, dans le

48. Ce discours est très peu cité ou reproduit. Pluchon en cite des extraits, op. cit., pp. 374-376 ; pour la version complète voir, notamment, Collection Victor Schœlcher, $V$, Haïti, B.N.F, Départ. des Manuscrits, n.a.f. 3633.

49 . L'article 68 de la constitution stipule : “ toute personne a la faculté de former des établissements particuliers d'éducation et d'instruction pour la jeunesse sous l'autorisation et la surveillance des administrations municipales". Non seulement l'éducation est laissée aux particuliers, la structure sociale fondée sur le travail de la terre, le refoulement qu'elle suppose ne laisse pas voir comment la majorité transformée en cultivateurs peut avoir accès à ce savoir. On continuera donc à les maintenir en dehors des champs du savoir et à légitimer le pouvoir des élites minoritaires par ce même savoir. 
discours et la démarche de Toussaint ${ }^{50}$ ? Si cette dernière affirmation est juste (l'interprétation qu'en a donnée son auteur mise à part), il faut en déduire que le nouveau projet de société a été élaboré dans une tension idéologique, dont chacun des termes sera exploité par l'une et l'autre des deux branches de la nouvelle élite.

Cette tension n'a pas empêché une glorification de Toussaint. Mais quand en l'absence de Noirs à l'assemblée, il est encensé comme un Noir exceptionnel, on peut se demander qu'est-ce qui se jouait derrière ce culte de l'exception ? En tout cas, cette révérence n'est pas synonyme de concession absolue : tous les voeux du général en chef n'ont pu être satisfaits. En le précisant les constituants montrent qu'ils se sont efforcés de trouver des limites au pouvoir de Toussaint. Ils se justifient en recourant à l'idée de "l'amélioration progressive ". Leur insistance sur ce point semble viser aussi un autre objectif : le contrôle social. C'est le sort des travailleurs, enfin de compte, qui mérite d'être "amélioré ". La constitution en définit le cadre légal, mais les acquisitions devront être progressives. L'argument de la perfectibilité semble trahir une inquiétude relative à d'éventuelles contestations sociales. En tout cas, il semble confiner dans une juste mesure les

50. Dorsinville, acteur politique et théoricien, dans les années 1940, du noirisme (idéologie de couleur qui s'opposait au mulâtrisme) en venait à dénoncer " l'imposture de 46 ". C'est à l'occasion de cette autocritique qu'il situe l'origine de cette idéologie reprise au $\mathrm{XIX}^{\mathrm{e}}$ siècle sous le slogan le pouvoir au plus grand nombre, dans la démarche se Toussaint. Il écrit “Il n'y avait rien de commun, entre cet homme [Toussaint] et ceux qui s'étaient donnés à lui comme "clientèle naturelle ", que la couleur de leur peau. Par où l'on voit que les logiques semblent inversées, puisqu'il faut écrire: " Tout les séparait, sauf la peau ". Mais, aussi par où concevoir que cette peau, considérée comme symbole, était donc bien puissante, un accident tenant le rôle essentiel". L'auteur poursuit "Cet héritage, des générations l'avaient recueilli et transmis à ceux de 1946, dont le " noirisme des anges " avait mal lu l'histoire, d'où la confusion entre l'accident et l'essence, l'imposture". Roger Dorsinville, Marche Arrière II, Port-au-Prince, Editions des Antilles S.A., 1990, p. 228. Une question : peuton mettre en doute que Toussaint ait travaillé à rendre irréversible la liberté générale des esclaves ? Dès lors, jusqu'où tient l'analogie entre ses manipulations idéologiques et celles des hommes du XIX ${ }^{\mathrm{e}}$ siècle et des années 40 et 50 du XX $\mathrm{XX}^{\mathrm{e}}$ siècle. Certes, le modèle social auquel il a cru a très peu modifié les rapports sociaux après la liberté générale. Est-ce parce qu'il n'avait rien de commun avec la masse des cultivateurs ? Voir la lecture de Yves Bénot, op. cit. 
acquis de la liberté générale. Ce point est capital, puisqu'il touche à l'armature même du nouveau modèle social. Car toute la question réside dans le relèvement et le maintien de l'économie. Il convient de protéger la propriété et de garantir le travail dans la stabilité. Ainsi les esclaves transformés en cultivateurs doivent continuer à répondre aux exigences de l'économie de plantation. Leur statut a changé, mais ils sont refoulés et embrigadés sur les propriétés. Cela exigeait un dispositif autoritaire ${ }^{51}$. Cet ordre social est défini par les articles 13, 14, $15,16,17,18,75,76$ de la constitution.

Existe-t-il des fondements théoriques à ce nouvel ordre social ? A quelles sources idéologiques ont puisé ses concepteurs ?

Une des sources directes est sans conteste la Proclamation à faire aux esclaves révoltés dans les colonies françaises écrite par Julien Raimond en $1793^{52}$. Il s'agit d'une proposition de message à adresser aux esclaves pour les ramener à l'ordre selon un plan d'affranchissement progressif. L'esprit et la lettre de certains articles de la charte y sont déjà. Par exemple le caractère "sacré" et le principe de l'inviolabilité de la propriété établis par l'article 13 de la constitution sont déjà énoncés en ces termes : "La première chose que la loi exige dans l'état de liberté et de sociabilité, c'est le respect pour les personnes et les propriétés. Il faut donc vous accoutumer à les respecter envers les autres, si vous voulez en ressentir les heureux effets, quand vous arriverez à les réclamer pour vous ". Comme le fait la constitution, Raimond définit l'amour du travail comme la première des qualités "et la plus indispensable" de l'homme libre. Les articles $14,18,75,76$ de la constitution stipulent, respectivement :

Art.14. - La colonie étant essentiellement agricole, ne peut souffrir de la moindre interruption dans les travaux de ses cultures.

${ }^{51}$. Sur la militarisation du travail agricole voir l'article de Vertus Saint-Louis déjà cité.

52 . Julien Raymond [sic, ailleurs Raimond], colon de Saint-Domingue, Réflexions sur les véritables causes des troubles et des désastres de nos colonies, notamment sur ceux de Saint-Domingue avec les moyens à employer pour préserver cette colonie de la ruine totale; adressée à la Convention nationale, 1793, l'an second de la République, pp. 19-29. 
Art. 18. - Le commerce de la colonie ne consiste uniquement que dans l'échange, des denrées et productions de son territoire (...).

Art. 75. - Elle (la colonie) proclame que c'est sur le respect des personnes et des propriétés que reposent la culture des terres, toutes les productions, tout moyen de travail et tout ordre social.

Art.76. - Elle proclame que tout citoyen doit ses services au sol qui le nourrit ou qui l'a vu naître, au maintien de la liberté, de l'égalité, de la propriété, toutes les fois que la loi l'appelle à les défendre.

Voici ce qu'avait écrit le constituant quelques années plus tôt : " D'un autre côté, comme vous ne devez jamais oublier les bienfaits que vous recevez de la nation, vous devez dans tous les temps lui en témoigner votre reconnaissance ; et rien ne saurait mieux la lui prouver qu'en continuant toujours, par votre travail, à faire fructifier le sol des colonies, afin d'en obtenir une grande quantité de denrées, dont le produit tournera toujours à votre avantage; et remarquer que cette manière de témoigner à la nation de votre reconnaissance, sera pour vous une nouvelle source de bonheur; car, si vous négligez, après votre liberté obtenue, de cultiver les riches denrées des colonies, alors la nation n'ayant plus d'échanges à faire avec vous, elle ne porterait plus dans ces contrées tous les objets de nécessité et de commodité qui donnent les jouissances auxquelles vous aspirez".

Le rapport à la propriété et au travail formulé par Raimond (qui fréquentait le cercle des Amis des Noirs et les Jacobins) ne diffère pas des propositions émises par les abolitionnistes.

\section{DE SONTHONAX A TOUSSAINT : \\ LE MODELE NEOCOLONIAL ET L'IDEOLOGIE ABOLITIONNISTE}

Toussaint a entrepris de consolider la liberté générale. Consolider car celle-ci a été conquise sous la conduite d'autres chefs. Dès cette conquête, en 1793, les prémisses du modèle louverturien avaient été jetées. Elles sont contenues dans la réponse apportée par 
Sonthonax pour assurer la survie de la colonie. De ce point de vue, il y a plus de points communs entre Sonthonax et Toussaint qu'on ne le pense. Dans cette même perspective, il semble qu'il y a moins d'incohérence chez le premier que Pluchon ne l'écrit, et Toussaint paraît être moins "un révolutionnaire d'ancien régime" que cet historien veut le faire admettre. De l'un à l'autre il s'agissait d'apporter une solution à la question du rapport liberté/travail posée concrètement par la liberté générale. Problème éminemment préoccupant à l'époque pour la réflexion politique et économique, dont les principales propositions avancées inspireront Sonthonax et Toussaint. Ils ont, en effet, trouvé dans les nouvelles formulations du libéralisme les présupposés qui leur ont fait croire en la possibilité d'un nouveau modèle social post-esclavagiste : le modèle néo-colonial.

\section{Liberté et travail.}

\section{Le cadre conceptuel de la réponse de Sonthonax}

Michel Vovelle a rappelé récemment que "Sonthonax est à la fois un homme de conviction et un stratège qui, dans la limite même du cadre conceptuel qui est le sien*, va être amené à sortir de lui-même ; cet homme de loi, ce robin confronté à la réalité des choses, prit la stature de ceux par qui arrivent des proclamations propres à changer le monde. Par là-même, Sonthonax est véritablement le précurseur, celui qui va plus loin que les stratégies prudentes de la Société des amis des Noirs au-delà des solutions de compromis de l'affranchissement progressif. * A ce titre, Sonthonax mérite de triompher de l'oubli." 53

Ce dépassement, perçu positivement par Vovelle, n'est qu'un abus de pouvoir aux yeux de Pluchon. Celui-ci reconnaît que le Commissaire “n'avait pas été chargé de préparer l'abolition

53. A l'occasion d'un colloque tenu au Musée des Arts d'Afrique et d'Océanie les 7 et 8 septembre 1990, propos rapportés par Serge Barcellini (Président de l'association "Mémoire de L.-F. Sonthonax"), "Deux mémoires dans le temps présent : Léger Félicité Sonthonax Victor Schœlcher" in Les Abolitions de l'esclavage de L.F. Sonthonax à V. Schœlcher, 1793, 1794, 1848, textes réunis et présentés par Marcel Dorigny, Presses Universitaires de Vincennes et Editions Unesco, 1995, p. 390. 
progressive " ; "son initiative inattendue, explique-t-il, [... ] détruisit la domination des Blancs brutalement et non progressivement " ${ }^{\text {. }}$. Tout en appréciant différemment les faits Pluchon admet donc que le seul horizon possible était l'affranchissement progressif. Il introduit une autre appréciation concernant le cadre conceptuel. Il voit en Sonthonax un personnage "ambigu" qui le 5 mai recommande le maintien du code noir et le 29 août proclame la liberté générale. Cette ambiguiité n'est que "stratégie", rectifie-t-il, et la décision du commissaire apparemment "brutale”, en réalité, avait été longtemps préparée, dictée par un seul mobile : "la destruction de la caste des planteurs". Il en vient à se demander dans quelle mesure cet homme "sans rigueur dans la pensée, sans grandeur" obéit-il aux exigences des Lumières. Il répond: "Pour une part, certainement, mais aussitôt reniée par l'établissement d'un véritable Code républicain du servage, substitut $\mathrm{du}$ Code noir, qui accompagne la proclamation d'une liberté formelle " 55 . Pluchon présuppose là une homogénéité si ce n'est une univocité de la pensée des Lumières, notamment en ce qui concerne l'esclavage.

En fin de compte pour Vovelle comme pour Pluchon, au-delà de leur divergence dans l'appréciation des faits, Sonthonax se situe dans un horizon conceptuel bien circonscrit. Leur argumentation soulève un ensemble d'interrogations. La proposition des Amis des Noirs d'un affranchissement progressif constitue-t-elle les limites de leur cadre conceptuel ? La pensée des Lumières n'est-elle pas ambiguë sur le régime colonial et l'esclavage? Dans quelle mesure ce que Pluchon appelle la haine de Sonthonax pour les colons propriétaires ne s'applique qu'à lui seul ?

Bien avant la première mission de celui-ci à Saint-Domingue la réforme du régime colonial était à l'ordre du jour dans les milieux officiels. Des plaintes et des alarmes parvenues aux Bureaux des colonies avaient été enregistrées et méditées. Venant de sources diverses, les informations allaient toutes dans le même sens et avaient amené des esprits avertis à tirer les conclusions qui s'imposaient. "Les

\footnotetext{
${ }^{54}$. Pluchon, op. cit., p. 89.

${ }^{55}$. Ibid., p.91.
} 
colonies devaient être défendues contre les colons eux-mêmes*, explique Michèle Duchet, il fallait réformer le système esclavagiste, ou consentir à l'échec économique, à la faillite d'une politique coloniale. Ce n'était plus possible. Le commerce colonial tenait déjà une trop grande place dans l'économie bourgeoise, l'Etat se trouvait engagé dans une stratégie à l'échelle du monde des grandes découvertes, où les Antilles, les Mascareignes, les comptoirs africains jouaient un rôle essentiel. Aussi les instructions adressées aux gouvernements et aux intendants témoignent-elles d'une conscience de plus en plus nette des problèmes à résoudre, des mesures propres à éviter l'éclatement du système ${ }^{56}$." Après quelques tentatives de réforme, le courant réformiste a échoué. Sonthonax s'y est toutefois explicitement référé. Pluchon le reconnaît lui-même en affirmant qu'il réédite le programme du maréchal de Castries, lorsqu'il ambitionne (en janvier 1793) d'humaniser la condition des esclaves par la loi ${ }^{57}$. Le Commissaire, membre de la Société des Amis des Noirs, réaffirme ainsi la convergence existant sur ce point entre le courant réformiste et les abolitionnistes. Tous récriminaient la conduite des colons. Cette attitude n'est pas imputable au seul Sonthonax.

Les réformes étaient impératives parce que le système subissait des secousses internes qui laissaient croire possible son renversement par les esclaves. Une grande crainte avait gagné l'opinion française depuis les années vingt et trente du XVIII ${ }^{\mathrm{e}}$ siècle, alimentée par la relation des insurrections de la Jamaïque survenues dès 1720 ,

1734-1735, puis des événements de la Guyane ${ }^{58}$. Les troubles ont persisté sans qu'on ait pu en venir à bout. Tout annonçait que les chefs des premières rébellions allaient avoir des successeurs. Bon nombre d'observateurs le pressentaient et l'abbé Raynal l'exprima à travers l'image de la venue d'un "Spartacus nouveau, qui ne trouvera point de Crassus " 59 . Seize ans plus tard Sonthonax formulera aussi une "prédiction". L'exercice n'avait plus aucune valeur

56. Michèle Duchet, Anthropologie et histoire au siècle des Lumières (1971), Paris, Albin Michel, “Bibliothèque de l'Evolution de l'Humanité ”, 1995, p. 150.

57 . Pluchon, op. cit., p. 87.

58 . Michèle Duchet, op. cit., p. 143.

59. Ibid., pp. 170-177 
exceptionnelle. Mais il confirme, avant toute autre considération, qu'à part la proposition de l'affranchissement progressif et les tentatives de réformes déjà entreprises, la vision de Sonthonax était nourrie aussi des potentialités de la situation coloniale.

Que dit cette "prédiction"? Dans un article publié le 25 novembre 1790, dans le $n^{\circ} 63$ des Révolutions de Paris, Sonthonax écrit ceci : "Quant à la traite et à l'esclavage des nègres, les gouvernants de l'Europe auront beau résister aux cris de la philosophie, au principe de la liberté universelle qui germent et qui se propagent parmi les nations, qu'ils apprennent que ce n'est pas en vain que l'on montre la vérité aux peuples; que l'impulsion une fois donnée, il faudra absolument céder au torrent qui doit entraîner les anciens abus, et que le nouvel état des choses s'élèvera malgré les précautions qu'on prend pour en retarder l'exécution. Oui, nous osons le prédire avec confiance, un temps viendra, et le jour n'est pas loin où l'on verra un Africain à tête crépue, sans autre recommandation que son bon sens et ses vertus, venir participer à la législation dans le sein de nos assemblées nationales "60. Un tel propos, à cette date, n'était pas nouveau, cependant il avait encore une résonance révolutionnaire. En réalité la perspective reste purement réformiste et même conciliatrice : elle prévoit des nègres à l'assemblée nationale. Aucun catastrophisme à la Raynal qui avait averti que le "Code blanc sera terrible, si le vainqueur ne consulte que le droit des représailles. "61

$\mathrm{Au}$ moment où Sonthonax publie son article, l'abbé avait déjà renié ses positions radicales dans son Essai sur l'administration de Saint-Domingue, paru en 1785. L'Histoire des deux Indes elle-même, Michèle Duchet l'a fort bien montré, accumule, dans ses différentes éditions (1770, 1774, 1780), des propos contradictoires, dus à la multiplicité des sources, à son élaboration dans une phase de transition qui voit naître les projets d'affranchissement de Bessner et les oppositions de Malouet. Duchet relève que l'intérêt de l'ouvrage réside avant tout dans le fait qu'il reflète différents courants d'opinions. Les ambiguïtés, les contradictions, les volte-face de certaines personnalités

${ }^{60}$. Cité par Pluchon, op. cit., p. 86.

${ }^{61}$. Raynal, v, p. 288, cité par Duchet, op. cit., p. 175. 
témoignent des difficultés causées à la pensée des Lumières par la question de l'esclavage. Ce malaise a été fortement ressenti dans les débats à l'assemblée nationale de 1788 à 1791, il est manifeste dans les embarras des Amis des Noirs ou le fameux silence de Mirabeau au moment du rapport de Barnave ${ }^{62}$.

Que Sonthonax, à Saint-Domingue, ait pu, contrairement à son article de 1790, recommander le maintien du code noir dans un premier temps, avant de proclamer la liberté générale, cela ne semble pas relever d'une ambiguïté qui lui soit particulière. De plus, face à l'accession irréversible des esclaves à la liberté, il vivait l'accomplissement d'un événement qui, pour lui (comme pour d'autres) était dans l'ordre du possible. Comme dans son horizon conceptuel une colonie sans esclavage était réalisable et les conditions de la liberté définies, sa décision pouvait être prise. Les abolitionnistes et les physiocrates avaient soutenu qu'une colonie transformant ses esclaves en travailleurs libres connaîtrait une grande stabilité et une meilleure productivité. On comprend pourquoi la liberté ayant été conquise par les esclaves, la préoccupation de Sonthonax a été de réglementer le travail. D'où les règlements de culture.

Le Commissaire a mis en application les nouvelles idées émises par la pensée libérale.

\section{Les références de Toussaint}

Le 20 juin 1795, Tousssaint écrit à Laveaux : “ Je suis occupé à faire rassembler les cultivateurs, les conducteurs et les gérants pour les exhorter à l'amour du travail, qui est inséparable de la liberté ". Pluchon commente : "Il prend à son compte le sophisme, généreusement répété par Sonthonax et Laveaux : les plantations feront autant de revenu que par le passé parce que "l'homme libre, qui n'a rien, connaît le besoin du travail, le fait avec plus de patience et de

62. Jacques Thibau, op. cit., pp. 193-200. 
satisfaction lorsqu'il est sûr de retirer le fruit de ses travaux" ",63. L'auteur qualifie de sophisme le discours qui essaie d'articuler le nouveau rapport au travail, dont les sources théoriques sont tout à fait claires. Le statut de sophisme attribué aux propos de Sonthonax et de Laveaux lui laisse la main libre d'affirmer que le général Noir " ne partage [pas] les idées de son temps". C'est qu'il entend figer le portrait de Toussaint dans le moule de l'ancien régime. Il y voit un homme pratique, dont l'action n'est sous-tendue par "aucune philosophie "; un homme qui, face à son échec, en désespoir de cause sera "obligé de se rabaisser au niveau d'un despote africain "64. Les Lumières ne pouvaient en aucun cas accoucher d'un tel monstre...

Toussaint, pourtant, partageait réellement l'orientation prise par Sonthonax, par conviction personnelle. On s'en rend compte par le culte qu'il vouait à l'abbé Raynal, celui de l'Histoire des Indes. A ce propos, un témoignage du naturaliste français Descourtilz: "Je lui vis, dit-il, en peu de mots exposer verbalement le sommaire de ses proclamations, rétorquer les phrases mal conçues, mal saisies ; faire face à plusieurs secrétaires qui alternativement présentaient leur rédaction; en faire trancher des périodes sans effets ; transposer des membres pour les mieux placer ; enfin se rendre digne du génie naturel annoncé par Raynal, dont il révérait la mémoire, en l'honorant comme son précurseur. Le buste de cet auteur était respectueusement conservé dans chacun des cabinets particuliers attachés aux diverses résidences de cet Africain présomptueux. "65

Toussaint a eu pour référence philosophique et idéologique l'Histoire des deux Indes de Raynal. Une étude systématique sur sa connaissance de l'ouvrage, à travers ses écrits, serait à faire. Mais certaines indications laissent croire qu'il ne s'y est pas référé uniquement parce qu'il se serait identifié au Spartacus Noir. D'ailleurs s'il a lu le passage (intégré dans l'édition de 1774) où Raynal en appelle à la venue de ce héros, il a du coup pris connaissance des événements de la Guyane, cadre historique des propos de l'auteur.

\footnotetext{
${ }^{63}$. Pluchon, op. cit., pp. 135-136.

${ }^{64}$. Ibid, p. 444.

${ }^{65}$. Cité par Pluchon, op. cit., p. 336
} 
"Déjà se sont établies deux colonies de nègres fugitifs, que les traités et la force mettent à l'abri de vos attentats, raconte Raynal. Les éclairs annoncent la foudre, et il ne manque aux nègres qu'un chef assez courageux, pour les conduire à la vengeance et au carnage ". La connaissance de tels faits et l'appréciation de l'auteur qui les a rapportés n'étaient pas sans intérêt pour Toussaint. Il a su en tirer des leçons comme il l'a laissé comprendre à propos de la situation à la Jamaïque. Dans un rapport énergique envoyé aux Directeurs, après l'expulsion de Sonthonax (août 1797), dont il a publié un extrait sous le titre de Réfutation de quelques assertions d'un discours prononcé le 10 prairial an V par Viénot-Vaublanc, il prévenait "... si les projets du citoyen Vaublanc pouvaient avoir une quelconque influence sur le gouvernement français, qu'il se souvienne qu'il existe, dans le sein de la Jamaïque, sur les Montagnes Bleues, un petit nombre d'hommes assez jaloux de leur liberté, pour avoir forcé jusqu'à ce jour l'orgueil et la puissance anglaise à respecter des droits qu'ils tiennent de la nature, et que la constitution française nous garantit ",66. Les événements de la Jamaïque, on l'a vu, ont joué un rôle important sur les idées abolitionnistes et le courant réformiste. Ils ont été relatés en France par Prévost (Pour et Contre, en 1735, à plusieurs reprises - journal tomes IV, V, VI, et dans les Voyages de Lade, 1744) pour les insurrections de 1720 et de 1734-1735), par Raynal, pour la période $1739-1770^{67}$. Toussaint n'a-t-il pas médité la figure de Moses Boom Sam, chef des marrons de la Jamaïque qui se présentait comme le Moïse du peuple africain, son guide et son libérateur ? Il y a aussi le cas des nègres du Surinam auquel les Hollandais ont accordé l'indépendance, fait rapporté dans l'édition de 1780 de l'Histoire des deux Indes.

L'affirmation de Raynal du droit de l'esclave à la révolte se fondait sur des données historiques précises. La connaissance de ces faits ne pouvait que renforcer la détermination de Toussaint dans sa lutte. Le futur gouverneur de Saint-Domingue avait à sa disposition, consignés dans les trois éditions de l'ouvrage de Raynal (à supposer

${ }^{66}$. Ibid., pp.197-198.

${ }^{67}$. Histoire des deux Indes, 1780, in $8^{\circ}$, tome vii, pp. 59 et 65 . 
qu'il les avait toutes) "un éventail complet des solutions et des réformes, qui entre 1770 et 1780, furent successivement proposées ou soutenues par la partie la plus éclairée de l'administration et par les physiocrates $^{68}$ ".

Les références théoriques de Toussaint étaient les mêmes que celles de Sonthonax. Lui aussi, se basant sur le discours des abolitionnistes et des physiocrates, croyait politiquement et économiquement viable un nouvel ordre social fondé sur le maintien de la grande plantation exploitée par des travailleurs libres.

\section{DE SAINT-DOMINGUE A HAÏTI : \\ LE MAINTIEN DU CONTRAT DE 1801 ET DU MODELE NEOCOLONIAL}

De Sonthonax à Toussaint, on a assisté aux péripéties de la mise en place du modèle néocolonial. Après la tentative avortée de Bessner en Guyane, Toussaint est peut-être celui qui est allé le plus loin, à l'époque, dans l'expérimentation de cette voie. Sans doute y croyait-il, cependant il en était encore à une phase expérimentale au moment de son arrestation et de sa déportation. Le modèle n'avait pas encore atteint un certain degré de maturation, de stabilité, d'équilibre, d'autorégulation.

Il ne s'y était pas trompé en se persuadant que les acquis étaient constamment menacés, que la métropole organisait le retour à l'ordre esclavagiste. Il préparait depuis longtemps la résistance armée. Les représentants de la métropole l'avaient d'ailleurs bien ressenti. Le désarmement des cultivateurs fut l'une des principales tâches confiées à Hédouville. Ce fut l'une des principales préoccupations de Leclerc. La militarisation est la clé de voûte de la stratégie de Toussaint. Devenu gouverneur il consacre une importante enveloppe budgétaire à son armée. Il avoue même avoir consacré ses propres fonds aux besoins de cette institution. Il s'est employé à créer les conditions d'une lutte acharnée contre le retour à l'ordre ancien. Une guerre totale

${ }^{68}$. Michèle Duchet, op. cit., p. 171. 
(guerre classique, guerre populaire, guerre bactériologique naturelle) attendait Leclerc. Ce fut la guerre de l'indépendance.

Toussaint a inventé la formule de l'Etat-associé. Ce régime politique a avorté, mais l'Etat indépendant en a maintenu le modèle socio-économique qui le sous-tendait. Comme si l'indépendance avait aménagé le contexte politique favorable à l'application du projet louverturien. Depuis, la structure sociale et économique est maintenue et les élites ont expérimenté divers régimes politiques.

Claude Moïse a constaté un moment d'hésitation après la proclamation de l'indépendance. "Il ne semble pas que les dirigeants aient senti la nécessité de doter le nouvel Etat d'une Constitution. Les premiers textes sont muets sur le régime politique. De la reddition de Rochambeau en novembre 1803 jusqu'à la charte de 1805, c'est le vide constitutionnel. Ce silence paraît traduire les hésitations de la classe dirigeante quant à la gestion de l'héritage et à l'avenir économique de la nouvelle Nation. Celle-ci s'en remet à Dessalines." ${ }^{\text {"69 }}$. Est-ce bien une hésitation... ? N'est-ce pas plutôt l'expression d'une continuité ? La proclamation des Généraux ${ }^{70}$ qui nomme Dessalines gouverneur général à vie le $1^{\text {er }}$ janvier 1804 reprend les mêmes termes par lesquels la constitution de 1801 confiait le pouvoir à Toussaint. Comme s'il s'agissait d'un simple transfert de l'un à l'autre dans des conditions exceptionnelles. Cette passation des pouvoirs semble être conforme à l'article 33 de la constitution de 1801 qui prévoyait la procédure de nomination d'un nouveau gouverneur en cas de vacance. Dessalines le plus haut gradé, a recueilli le pouvoir, puis est nommé par les Généraux convoqués à cet effet, selon les prescriptions. Il ne manquait que l'assemblée centrale, inexistante.

L'élaboration d'une nouvelle constitution sera avant tout un nouvel aménagement ou une nouvelle définition du régime politique. Du régime impérial de Dessalines au régime républicain de Pétion et de Boyer, tout le dispositif légal et administratif a été réaménagé

${ }^{69}$. Claude Moïse, op. cit., pp. 29-30.

${ }^{70}$. Acte des Généraux nommant Dessalines Gouverneur Général à vie, in Linstant de Pradines, Recueil des lois et actes du gouvernement d'Haïti, tome $1^{\text {er }}$, 1804-1805, $2^{\mathrm{e}}$ édition, Paris, A. Durand-Pédonne-Lauriel, 1886, p. 5. 
autour du modèle social à l'essai sous Toussaint. D'où l'insistance sur l'inviolabilité de la propriété privée ; sur la nécessité de la culture, base de la prospérité de l'Etat, par le biais du commerce. Les constitutions de 1805 (déclaration préliminaire, dispositions générales, art.6, 21), 1806 (art. 3, 7, 8, 22, 23), 1816 (art. 6, 10, 11, 26, 27) le réaffirment sans ambages. L'article qui définit le système social en 1801(art. 75) a été repris tant en 1805 qu'en 1806 (art. 22) et en 1816 (art. 26). Il fait reposer tout l'ordre social sur le respect des personnes et des propriétés. Cette structure sociale maintenue de Toussaint à Pétion sera systématisée sous Boyer à travers la série des codes, dont le code rural (1826) forme achevée des différents règlements de culture pris jusqu'à Pétion.

La société haïtienne s'est consolidée à partir du modèle néocolonial. Il devient dès lors difficile d'admettre la thèse selon laquelle Haïti a glissé dans le néocolonialisme à partir de la dette de l'indépendance ${ }^{71}$, bien que le paiement de cette dette ait eu des conséquences néfastes pour l'économie du pays.

D'abord, ce que les théories du sous-développement allaient appeler l'échange inégal était déjà au cœur même du système colonial. "En effet, le négoce métropolitain vend très cher les produits européens, farines, vins, ferrements, et les esclaves africains ; et il achète les denrées coloniales, sucre, café, indigo, coton, au moindre prix. Pour faire face à cette manœuvre, les propriétaires empruntent aux armateurs et aux commerçants qui outre leur rôle, assument le rôle de banquiers, tout comme en France" ${ }^{, 72}$. Maintenir le mode de production coloniale revenait à garder la même place dans cette structure d'échange et à continuer d'en subir les conséquences. Quand on compare le tableau des importations et des exportations de Saint-

71. Thèse soutenue par Benoît Joachim, op. cit. ; depuis, admise et fréquemment reprise. Récemment André Corten “L'Etat faible haïtien, économie et politique " in Laennec Hurbon, op. cit.(colloque de Port-au-Prince), p. 290.

${ }^{72}$. Pluchon, op. cit., p. 33. Voir aussi l'article de Vertus Saint-Louis cité. 
Domingue et d'Haïti jusqu'à Boyer on constate que la configuration demeure la même ${ }^{73}$. Aucun changement notable.

La fabuleuse richesse de Saint-Domingue à la fin du XVIII ${ }^{\mathrm{e}}$ siècle obnubilait les élites haïtiennes. Celles-ci croyaient pouvoir redonner à Haïti sa "splendeur d'antan" en la circonscrivant dans son rôle de pourvoyeuse de matière première et surtout de produits tropicaux, coloniaux. Elles ont ainsi gardé et raffermi les liens de la dépendance. Certains penseurs du $\mathrm{XIX}^{\mathrm{e}}$ siècle avaient compris que le pays devait s'engager dans la voie de l'industrialisation. Aucun groupe social suffisamment fort n'a émergé pour infléchir dans ce sens la politique des gouvernements.

\section{CONCLUSION}

Le contrat social de 1801 n'a pas été renégocié en 1804. La structuration sociale haïtienne découle du modèle néocolonial qui en est issu. Ce modèle a installé Haïti dans la dépendance et le sousdéveloppement en générant un Etat autoritaire fondé sur l'exclusion des masses. Il s'inscrit dans un horizon théorique : la pensée des abolitionnistes et des physiocrates. Ceci éclaire la ressemblance observée entre Haïti et les autres pays qui n'ont pas fait de révolution anti-esclavagiste. Quand de 1793 à 1801 les idées abolitionnistes s'appliquaient à Saint-Domingue, elles étaient encore inacceptables. Elles avaient encore une dimension révolutionnaire dans une Europe persuadée que sa richesse reposait sur l'esclavage. Il a fallu attendre la

73. Pour l'état des importations et des exportations en 1789, voir T.C. Mozard, Etat des importations et exportations du commerce de France, Paris, an II ; voir M. Wante, Importance de nos colonies, particulièrement celle de Saint-Domingue..., Paris, chez Ballard,1805, Annexes 1, 2, et 3 ; Pour la période 1802-1803 on peut consulter l'état des mouvements du port publiés dans les Affiches Américaines de Saint-Domingue... Le Cap Français et La Gazette officielle de Saint-Domingue.... Le Cap Français, BNF, Res. Fol. LC12. 30. Pour la période haïtienne jusqu'à Boyer, voir Linstant Pradines, Recueil général ..., les “ lois relatives aux droits de douane”, t. 1 à 6. 
deuxième moitié du $\mathrm{XIX}^{\mathrm{e}}$ siècle pour que s'exécute la valse des abolitions. Les solutions alors adoptées ressemblent à celles appliquées de Sonthonax à Boyer en passant par Toussaint, le problème étant de maintenir la structure économique coloniale en convertissant les esclaves en travailleurs libres. Il a donc fallu du temps et surtout des transformations dans l'économie des métropoles pour que celles-ci saisissent les vertus du projet abolitionniste qui formulait un nouvel aménagement du rapport colonie/métropole. En ce sens, le discours abolitionniste est l'énoncé systématique de l'idéologie néocolonialiste.

Le régime colonial avait pris aussi du temps à s'installer. Le contrat colonial entre la France et Saint-Domingue a été mis au point entre l'appel des ressortissants français de la Tortue en 1640 et la publication du code noir en 1685. La rupture de ce contrat amorcé en 1790 (le décret du 8 mars / la déclaration de 25 mai de l'assemblée de Saint-Marc) s'est concrétisée en 1801. Pendant près de cent soixante et un ans la colonie a vécu sous ce contrat. Celui-ci a défini un cadre dans lequel s'est développée la colonie. Le modèle s'est dynamisé tout en connaissant divers mouvements de contestations (révoltes blanches, résistances multiformes des esclaves...) jusqu'à l'élaboration du nouveau contrat.

Le modèle néocolonial à l'essai depuis 1793 est formalisé en 1801 et sa phase de consolidation a débuté après 1804. Il a défini le cadre de développement de la société haïtienne. Il a survécu à de violentes remises en question et a connu des transformations jusqu'aux remises en causes en 1986. Onze ans après cette date l'alternative ne semble pas avoir été encore trouvée. 


\section{REFERENCES BIBLIOGRAPHIQUES}

Affiches Américaines de Saint-Domingue... Le Cap Français. (1802-1803 ; BNF, Res. Fol. LC12. 30.)

ARdouin Beaubrun, Géographie de l'île d'Haïti, Port-au-Prince, 1832.

ARNAUD André-Jean (dir.), Dictionnaire Encyclopédique de théorie et de sociologie du droit, Paris, L.G.D.J, 1993 (2 $2^{\mathrm{eme}}$ édition corrigée et augmentée).

BADIOU Alain, "Nous pouvons redéployer la philosophie", Entretien avec Roger-Pol Droit, Le Monde, 31 août 1993, recueilli dans Les grands entretiens du Monde, t.2, mai 1994.

BARTHElemy Gérard et GiRAult Christian (dir.), La République haïtienne. Etat des lieux et perspectives, Paris, ADEC-Karthala, I993.

CARTEAuX Félix, Histoire des désastres de Saint-Domingue. Soirées Bermudiennes ou entretiens sur les événements qui ont opéré la ruine de la partie française de l'île St-Domingue, Bordeaux, Pelletier-Lawalle, 1802.

Chemins Critiques, Revue Haïtiano-Caraïbéenne, Port-au-Prince, Vol. 2, $\mathrm{n}^{\circ} 3$, mai 1992 ; Vol. 3, $\mathrm{N}^{\text {os }} 1-2$, décembre 1993.

DEBIEN Gabriel, Esprit colon et esprit d'autonomie à Saint-Domingue au XVIII siècle, Paris, 1954

DoRIGNY Marcel, Les Abolitions de l'esclavage de L.F. Sonthonax à V. Schoelcher, 1793, 1794, 1848, Presses Universitaires de Vincennes et Editions Unesco, 1995.

DORSINVILLE Roger, Toussaint Louverture, Montréal, Port-au-Prince, CIDIHCA, 1987.

Dorsinville Roger, Marche Arrière II, Port-au-Prince, Editions des Antilles S.A., 1990.

DuCHET Michèle, Anthropologie et histoire au siècle des Lumières (1971), Paris, Albin Michel, “Bibliothèque de l'Evolution de l'Humanité ”, 1995.

Du MORIER Dumorier, Sur les Troubles des colonies et l'unique moyen d'assurer la tranquillité, la prospérité et la fidélité de ces dépendances de l'empire, en réfutation des deux discours de M. Brissot, des $1^{\text {er }}$ et 3 décembre 1791, Paris, Imp. de Didot Jeune, décembre 1791.

Frostin Charles, Les révoltes blanches à Saint-Domingue aux XVII ${ }^{E}$ et XVIII $I^{e}$ siècles, Paris, Editions de l'Ecole, 1975.

GISLER Antoine, L'esclavage aux Antilles françaises (XVII ${ }^{E}-X_{X}{ }^{E}$ siècles). Contribution au problème de l'esclavage (1965), Paris, Karthala, 1981 (nouvelle édition revue et corrigée).

GLISSANT Edouard, Monsieur Toussaint (1961), (version scénique), Paris, Seuil, 1986. 
Hector Cary, MoÏse Claude, Ollivier Emile, 1946-1976. Trente ans de pouvoir noir en Haïti. Tome premier / L'explosion de 1946. Bilan et perspectives, Québec, Collectif Paroles, 1976.

HuRbon Laënnec (dir.), Les transitions démocratiques. Actes du colloque international de Port-au-Prince, Haïti, Paris, Syros, 1996.

JANVIER Louis Joseph, Les constitutions d'Haïti (1801-1885), Paris, C. Marpon et E. Flammarion, 1886.

JOACHIM Benoît, Les racines du sous-développement en Haïti, Port-au-Prince, Imprimerie H. Deschamps, 1979.

L'Ami des Patriotes, N XVI, Imp. de Demonville, 1792 (Extrait). (BNF, LK 9 191).

De LACroix Pamphile (Général), La révolution de Haïti (1819), édition présentée et annotée par Pierre Pluchon, Paris, Karthala, 1995.

La Gazette officielle de Saint-Domingue... "Le Cap Français. (1802-1803)" ; BNF, Res. Fol. LC12. 30.)

MoÏsE Claude, Constitutions et luttes de pouvoir en Haïti, Montréal, CIDHICA, 1988, t. I.

PeYtraud, Histoire de l'esclavage aux Antilles françaises avant 1789, Paris, 1897.

PIERRE-CHARles Gérard, Vision contemporaine de Toussaint Louverture, Portau-Prince, CRESFED, 1992.

Placide-Justin Michel, Histoire politique et statistique de l'Ile d'Haïti, SaintDomingue, Paris, Briere Libraire, 1826.

PluChON Pierre, Toussaint Louverture. Un révolutionnaire noir d'Ancien Régime, Paris, Fayard, 1989.

De Pradines Linstant, Recueil des lois et actes du gouvernement d'Haïti, tome $1^{e r}, 1804-1805,2^{\mathrm{e}}$ édition, Paris, A. Durand-Pédonne-Lauriel, 1886 ; t. II à VI, Paris, 1861-1881.

RAIMOND Julien, Mémoire sur les causes des troubles et des désastres de la colonie de Saint-Domingue, Paris, Imp. du Cercle social, 1793.

RAIMOND Julien, Réflexions sur les véritables causes des troubles et des désastres de nos colonies, notamment sur ceux de Saint-Domingue avec les moyens à employer pour préserver cette colonie de la ruine totale; adressée à la Convention nationale, 1793.

SAINT-LouIs Vertus, "Régime militaire et règlement de culture ", Chemins Critiques, vol. 3, ${ }^{\text {os }} 1-2,1993$, pp. 183-227.

SCHELCHER Victor, Colonies étrangères et Haïti. Résultats de l'émancipation anglaise, t. 2, Paris, Pagnerre, 1843.

ThiBAu Jacques, Le temps de Saint-Domingue. L'esclavage et la Révolution française, Editions JC Lattès, 1989.

Trouillot Michel-Rolph, Les racines historiques de l'état duvaliérien, Portau-Prince, Editions Deschamps, 1986.

WANTE, Importance de nos colonies, particulièrement celle de SaintDomingue..., Paris, Ballard,1805. 


\section{Résumé}

L'anthropologue Michel Rolph Trouillot a affirmé récemment qu'il n'y a jamais eu de contrat social haïtien.

Si le contrat se comprend comme la formalisation d'un modèle social négocié entre des forces politiques à un moment donné, on peut soutenir qu'il y en a un. Il est antérieur à 1804. Il date de 1801, c'est-à-dire de la Constitution de Toussaint Louverture. Il formalise un modèle social à partir duquel s'est structurée la société haïtienne. Ainsi, resurgit la question de la nature de la rupture que constitue l'indépendance. Se pose aussi le problème de la nature du pouvoir louverturien dont la représentation habituelle ne laisse aucune place à l'idée de négociation.

Le modèle social louverturien, néo-colonial, s'inscrit dans un horizon théorique : la pensée des abolitionnistes et des physiocrates.

Maintenu après l'indépendance par les élites haïtiennes, il a installé Haïti dans la dépendance, en générant un Etat autoritaire fondé sur l'exclusion des masses.

\section{Abstract}

The anthropologist Michel-Rolph Trouillot has recently argued that there has never been a Haitian social contract. However, if by "contract" we mean the formalization of a social model negotiated between political forces at a given moment, we can affirm that a social contract has and does exist in Haiti. Its existence, however, dates not from the moment of Haitian independence in 1804, but from Toussaint Louverture' 1801 constitution, which formalized the model upon which Haitian society has been structured since. By examining the importance of this constitution, this article poses the question of the nature of Louverture's power, which is usually represented simply as an authoritarian system as opposed to one negotiated with the social forces of revolutionary SaintDomingue. Louvertures' neocolonial social model was inscribed in the context of the abolitionist and physiocratic thought of the $18^{\text {th }}$ century. Maintained, after independance, by the Haitian elites, it made Haiti economically dependent and generated an authoritarian state founded on the exclusion of the masses.

\footnotetext{
Mots-clés

Abolitionniste(s) - Affranchissement - Code noir - Contrat social Constitution - Louverture, Toussaint Raimond, Julien - Saint-Domingue
} 